(e-migrinter

e-Migrinter

12 | 2014

Les chiffres de l'immigration : un regard critique sur les statistiques

\title{
Compter les migrations internationales? Quelques réflexions à propos des statistiques produites en France et en Amérique latine
}

\section{Françoise Dureau}

\author{
OpenEdition \\ Journals \\ Édition électronique \\ URL : https://journals.openedition.org/e-migrinter/396 \\ DOI : 10.4000/e-migrinter.396 \\ ISSN : 1961-9685 \\ Éditeur \\ UMR 7301 - Migrinter \\ Édition imprimée \\ Date de publication : 15 octobre 2014 \\ Pagination : 7-31 \\ ISSN : 1961-9685 \\ Référence électronique \\ Françoise Dureau, «Compter les migrations internationales? Quelques réflexions à propos des \\ statistiques produites en France et en Amérique latine », e-Migrinter [En ligne], 12 | 2014, mis en ligne le \\ , consulté le 20 mai 2021. URL : http://journals.openedition.org/e-migrinter/396 ; DOI : https://doi.org/ \\ 10.4000/e-migrinter.396
}


DossiER

\section{Compter les migrations internationales ? Quelques réflexions à propos des statistiques produites en France et en Amérique latine}

Françoise Dureau

C

ette contribution sera organisée en trois temps :

1) Pourquoi des " chiffres"? Ce qui renvoie à deux questions : Pourquoi compter? Compter pour comprendre et compter pour agir sur le phénomène lui-même ou sur ses effets ; Pour qui compter? Institutions nationales vs institutions locales (en particulier les institutions ayant en charge la gestion des métropoles).

2) Pourquoi des chiffres de « l'immigration » et donc exclure ceux de «l'émigration"?

Il semble paradoxal de ne compter que les entrées. Dans un certain nombre de cas, ce sont les effets

d'une émigration massive qui importent : au niveau national (Uruguay, Colombie) ou au niveau local (Bogota). J'évoquerai donc la mesure de l'émigration dans les recensements des pays latinoaméricains (Uruguay, Colombie, Chili).
3) Au-delà du sens de la migration (entrée-sortie), une question de fond se pose, celle de la notion de résidence, notion de base du rattachement des hommes aux lieux opéré par la statistique, en particulier dans les recensements. J'évoquerai cette question en considérant : d'abord la saisie de la résidence dans les recensements ; puis la saisie des systèmes résidentiels des individus à partir de quelques enseignements issus de deux projets de recherche $\left(\right.$ METAL $^{1}$ et MEREV $^{2}$ ) et troisièmement, les innovations développées en France dans « l'Enquête nationale Transport ".

\footnotetext{
${ }^{1}$ Programme «Mobilités entre métropoles européennes et recompositions des espaces de vie» (2007-2011), financement ANR, UMR Géographiecités et UMR Migrinter. Coordination de l'équipe Migrinter Christophe Imbert. Terrains: Berlin, Bruxelles, Lisbonne, Londres, Paris.

2Programme «Métropoles d'Amérique latine dans la mondialisation: reconfigurations territoriales, mobilité spatiale, action publique» (2007-2012) financement ANR-AIRD programme «Les Suds aujourd'hui ». Équipe pluridisciplinaire de vingt-sept chercheurs dont la moitié sont latino-américains, coordination Françoise Dureau. Terrains: Bogotá, São Paulo, Santiago du Chili.
} 
Cette réflexion s'appuie sur deux projets collectifs, menés respectivement en Europe et en Amérique latine, par des équipes pluridisciplinaires (géographes, démographes et spécialistes d'autres sciences sociales) et qui partagent la même posture générale : l'étude des pratiques de mobilités spatiales est menée non pas seulement pour elles-mêmes, mais aussi pour comprendre les dynamiques des grandes métropoles.

Mon propos tire également parti de différentes sources, qui présentent des réflexions sur la mesure des migrations et des autres formes de mobilité spatiale dans les recensements de la population, en particulier :

- des synthèses publiées par le Centre latinoaméricain et caribéen de démographie (CELADE) de Santiago, notamment celle présentée en 2008 par Juan Chackiel (2008) ; - deux rencontres organisées en 2004 à Nogent sur Marne, par Valérie Golaz et moi-même, dont les actes sont disponibles dans la collection «Les numériques du Ceped»: «La plurirésidence », UR 6 de l'INED et UR 13 de l'IRD (juin 2004) et "Mobilité et résidence», CEPED (novembre 2004).

\section{Des « chiffres »}

\section{Pourquoi compter?}

La production d'informations répondant à des finalités scientifiques compter pour comprendre - est marquée par la tension entre deux objets : comprendre les comportements individuels (approche démographique) ou les dynamiques des lieux (approche géographique). En outre, se pose la question des lieux : l'intérêt porte-t-il sur le pays ou la ville?

S'il s'agit de compter pour agir, ce peut être pour agir sur le phénomène luimême, c'est-à-dire "contrôler» la migration (entrées ou sorties); ou pour agir sur certaines conséquences du phénomène (par exemple, répondre à la demande de logement ou de services publics en matière d'éducation, de santé, de transport).

\section{Pour qui compter?}

Nous sommes dans une situation paradoxale: les systèmes nationaux de statistique restent complètement orientés vers la production d'informations pour des institutions nationales, alors que le contexte de décentralisation des pouvoirs et des compétences produit un besoin grandissant d'informations à un niveau local. Ainsi, on ne dispose que de très peu d'informations relatives, par exemple, aux mobilités internes aux grandes villes, qui concentrent pourtant une part croissante des populations - et des richesses - du monde.

Ce paradoxe se pose dans des termes particuliers si l'on considère la migration internationale. $\mathrm{La}$ production locale d'informations dans les grandes villes est très focalisée sur des phénomènes «locaux», dont la gestion relève directement de choix urbanistiques (les déplacements quotidiens intra-urbains par exemple). Elle ne prend que peu en considération un phénomène relevant d'une autre échelle, tel que celui de la migration internationale. Or, les métropoles sont des lieux importants de la migration internationale et des investissements internationaux et les «gouvernements métropolitains» ont un pouvoir important, tant en termes de ressources pour produire de l'information qu'en termes de définition de politiques spécifiques. Ce sont des acteurs «qui comptent» dans la production de l'information sur la migration internationale et dans la définition de politiques ou dans la mise en œuvre d'actions en direction des migrants internationaux pour attirer certains immigrants (élites) et capter, canaliser les investissements des émigrés. 


\section{Les chiffres de « l'immigration »}

Les situations migratoires diffèrent largement entre la France et l'Amérique latine. Cependant, en France comme en Amérique latine, on ne peut réduire la dynamique migratoire à l'arrivée et à l'installation d'immigrants.

Très schématiquement, l'Amérique latine est marquée par une migration vers les États-Unis et vers certains pays européens (en particulier l'Espagne, jusqu'à récemment) et l'importance des migrations entre pays de la région:

- existence de couples migratoires (Colombie-Venezuela, Bolivie-Argentine) ;

- processus d'attraction ou d'expulsion de certains pays, à certains moments, selon leur situation économique et politique (par exemple, le Venezuela);

- transit par le Mexique pour une partie de la migration vers les États-Unis.

En 2005, on estime à 3,3 millions le nombre de Colombiens résidant à l'étranger (pour une population de 45 millions d'habitants, soit $8 \%$ de la population totale $\mathrm{du}$ pays). Si l'on considère Bogota seulement, on compte $9 \%$ d'émigrants en 2005. L'histoire migratoire colombienne est marquée par plusieurs vagues d'émigration : dans les années 1960-1970, l'émigration, qui touche plutôt des secteurs populaires, est principalement dirigée vers le Venezuela et secondairement vers les États-Unis et Panama. Dans les années 1980, les ÉtatsUnis sont le principal lieu de destination de l'émigration depuis la Colombie. À partir des années 1990, on observe une diversification des flux d'émigration, en direction du Canada, de l'Australie et de l'Europe. La crise économique et politique de la fin des années 1990 se traduit par une intensification de l'émigration notamment des classes moyennes et supérieures vers les États-Unis, l'Espagne, le Venezuela et l'Équateur. L'émigration augmente très fortement: quasiment un million d'émigrants sont sortis de la Colombie entre
1995 et 2000. Les flux depuis Bogota se distinguent de ceux du reste du pays: l'émigration depuis la capitale colombienne est moins dirigée vers l'Espagne, et davantage vers les États-Unis, le Canada, l'Australie et le reste de l'Europe (hors Espagne).

En France comme en Amérique latine, l'enjeu réside dans la connaissance des mouvements internationaux de population qui touchent les territoires, de leur intensité, et de leurs caractéristiques :

- géographiques (vers/depuis où : quels pays, quelles localités, quels quartiers de métropoles ?) car raisonner de pays à pays est insuffisant ;

- temporelles (rythmes des circulations et des installations);

- et sociales (qui migre ?).

\section{Compter l'immigration dans les recensements latino-américains}

On retrouve en Amérique latine les questions classiques relatives :

- au lieu de naissance (et à la date d'arrivée dans le pays pour les nés à l'étranger) ;

- au lieu de résidence antérieur et à la durée dans la résidence actuelle ;

- au lieu de résidence X années avant le recensement (le plus souvent, cinq ans).

À partir de ces trois lieux, sont identifiées quatre situations individuelles :

- non migrant: les trois lieux sont identiques (ce qui ne signifie pas que les individus n'ont effectué aucune migration. Une émigration peut avoir été suivie d'une immigration);

- migrant ancien : le lieu de recensement est le même que le lieu occupé cinq ans auparavant, tout en étant différent du lieu naissance ;

- migrant récent: le lieu naissance est identique au lieu occupé cinq ans avant, mais différent du lieu de recensement ;

- migrant multiple: les trois lieux sont différents ; 
- migrant de retour : le lieu de naissance est celui du recensement, mais diffère du lieu occupé cinq ans avant.

Comme le note Juan Chackiel (2008) : "En el caso de la migración, se mide lo que se define $»^{3}$. Le découpage géographique et l'échelle temporelle (le nombre d'années entre les moments où l'on repère le lieu de résidence) ont un effet direct sur la mesure du phénomène. En outre, du fait de la mortalité, on ne mesure que les mouvements des personnes encore vivantes au moment du recensement. Au-delà de ces limites, bien connues, de la mesure de stock de migrants à partir des recensements, mention doit être faite d'une pratique de plus en plus courante dans les recensements: l'application $d u$ questionnaire complet seulement à un échantillon de la population. De ce fait, l'un des principaux avantages du recensement (la possibilité, liée à son exhaustivité, de produire des statistiques pour des unités spatiales de petite taille) peut être remis en cause. Il devient ainsi difficile de disposer d'informations à un niveau géographique très désagrégé.

Dans les figures qui suivent, nous montrerons une série de cartes issues de la question sur le lieu de résidence cinq ans avant le recensement: leur réalisation suppose l'accès à une information précisément localisée.
Les cartes de la figure $\mathrm{n}^{\circ} 1$ et celles des figures suivantes, ont été produites dans le cadre du projet METAL. L'ensemble des cartes réalisées pour les trois villes étudiées (Bogota, Santiago, São Paulo) à partir des deux derniers recensements disponibles dans chaque pays sont disponibles sur Internet ${ }^{4}$.

Ces premières cartes mettent en évidence la concentration des migrants récents dans certains lieux de la capitale du Chili :

- le centre, quartier de réception de la migration péruvienne et bolivienne, dans de l'habitat dégradé ;

- le «cône» du nord-est, qui concentre l'essentiel de l'habitat de haut standing, pour les migrants des pays développés.

On observe une segmentation des zones de réception de la migration internationale, selon le niveau socioéconomique des migrants. Chacun des flux s'insère dans des segments différents du marché du logement de la ville.

La figure $\mathrm{n}^{\circ} 2$ présente le même type de carte, mais cette fois-ci à Bogota, dans une ville où le poids de l'immigration internationale est bien moindre qu'à Santiago du Chili. Entre 1993 et 2005, on observe une réduction du poids de l'immigration internationale. Aux deux dates, les migrants sont concentrés dans les quartiers de haut standing, au nord-est de la capitale colombienne.
${ }^{3}$ « Dans le cas de la migration, est mesuré ce qui est défini » (traduction : Françoise Dureau).
${ }^{4}$ http://www.mshs.univpoitiers.fr/migrinter/metal/webmaps.html 
Figure $\mathbf{n}^{\circ} \mathbf{1}$ : Cartes des immigrants récents à Santiago du Chili (2002).

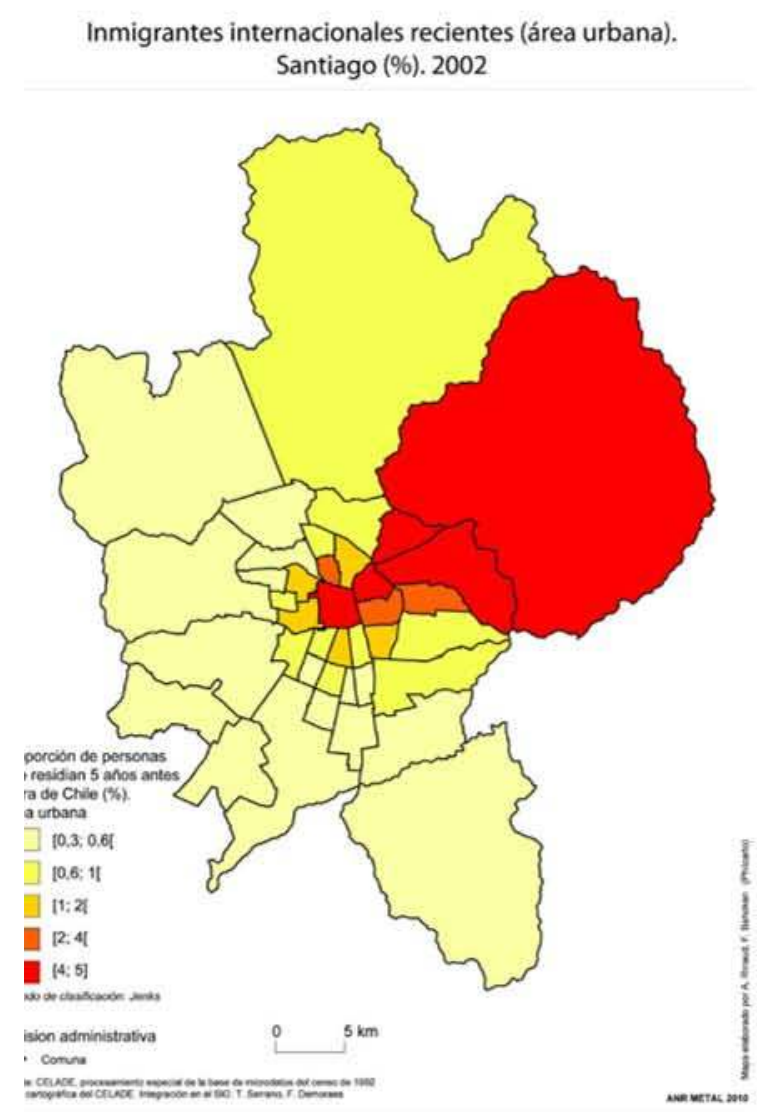

Toutes origines

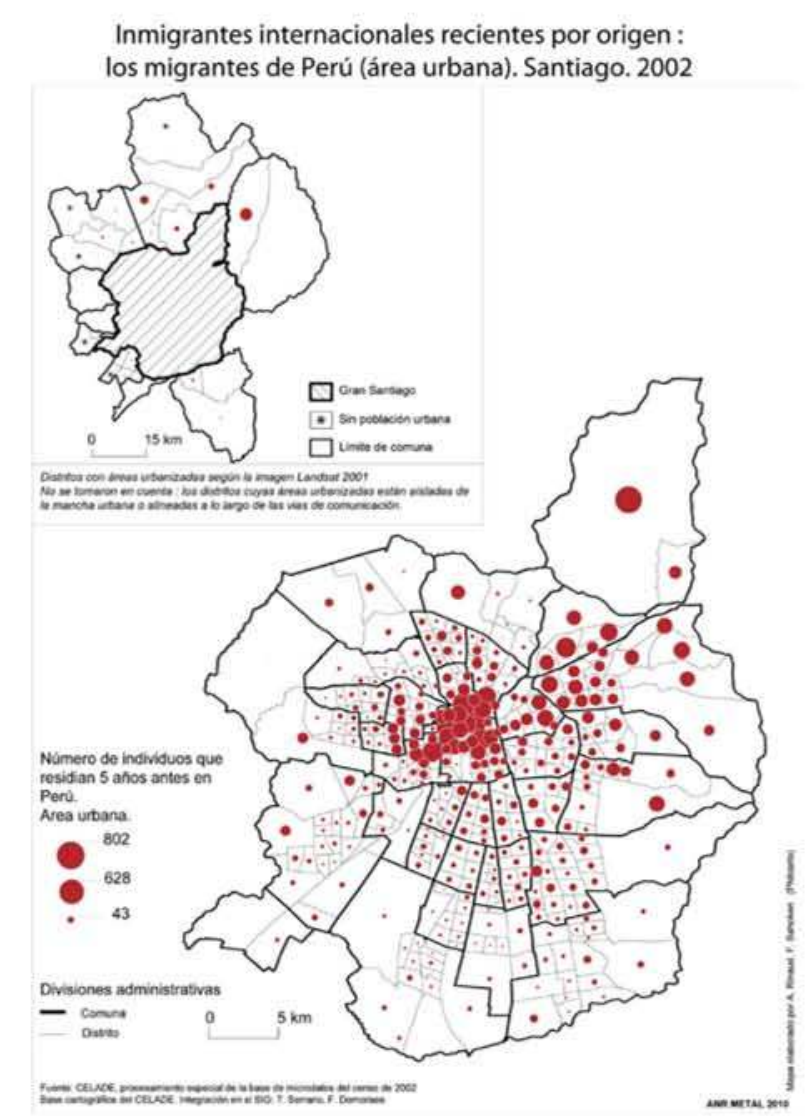

Pérou
Inmigrantes internacionales recientes por origen : los migrantes de paises desarrollados (área urbana).
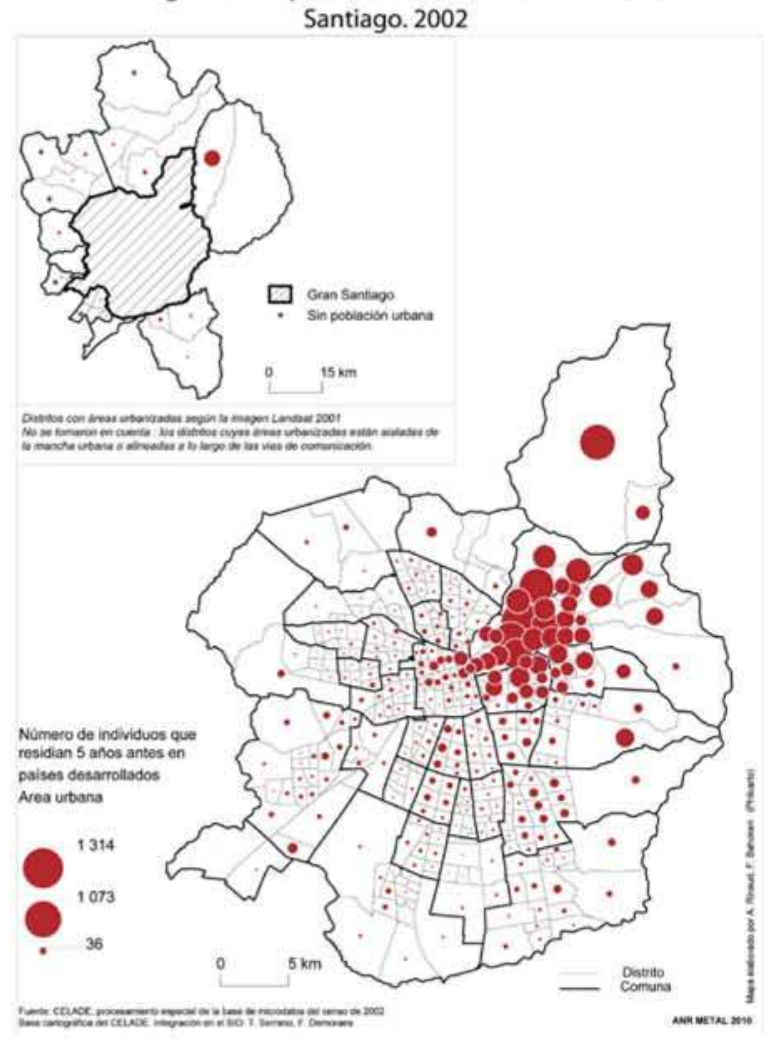

Amérique du Nord, Europe et autres pays développés 
Figure $\mathbf{n}^{\circ} \mathbf{2}$ : Cartes des immigrants récents à Bogota : 1993 et 2005. Inmigrantes internacionales recientes (área urbana). Bogotá (\%). 1993

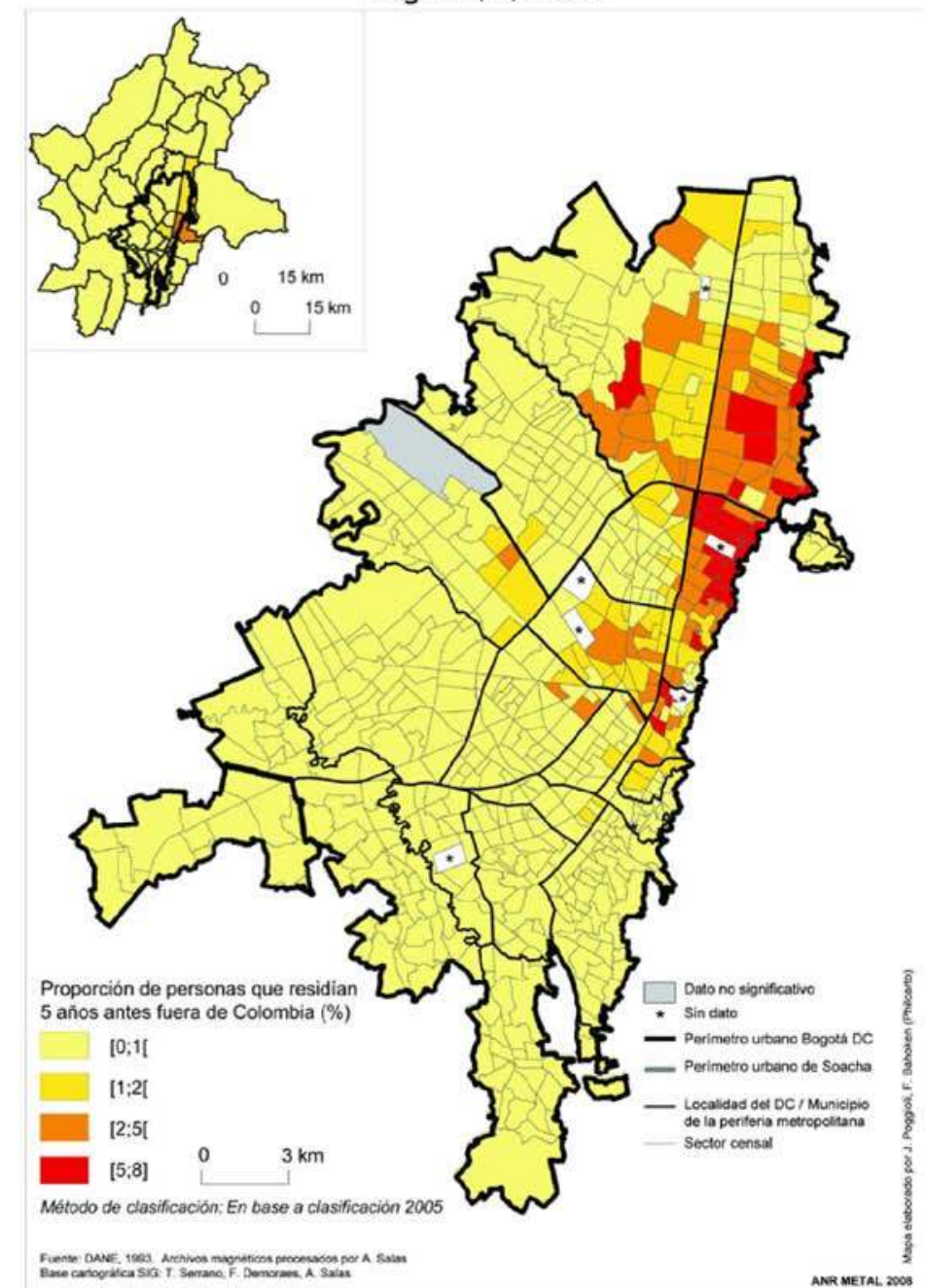

Inmigrantes internacionales recientes (área urbana).

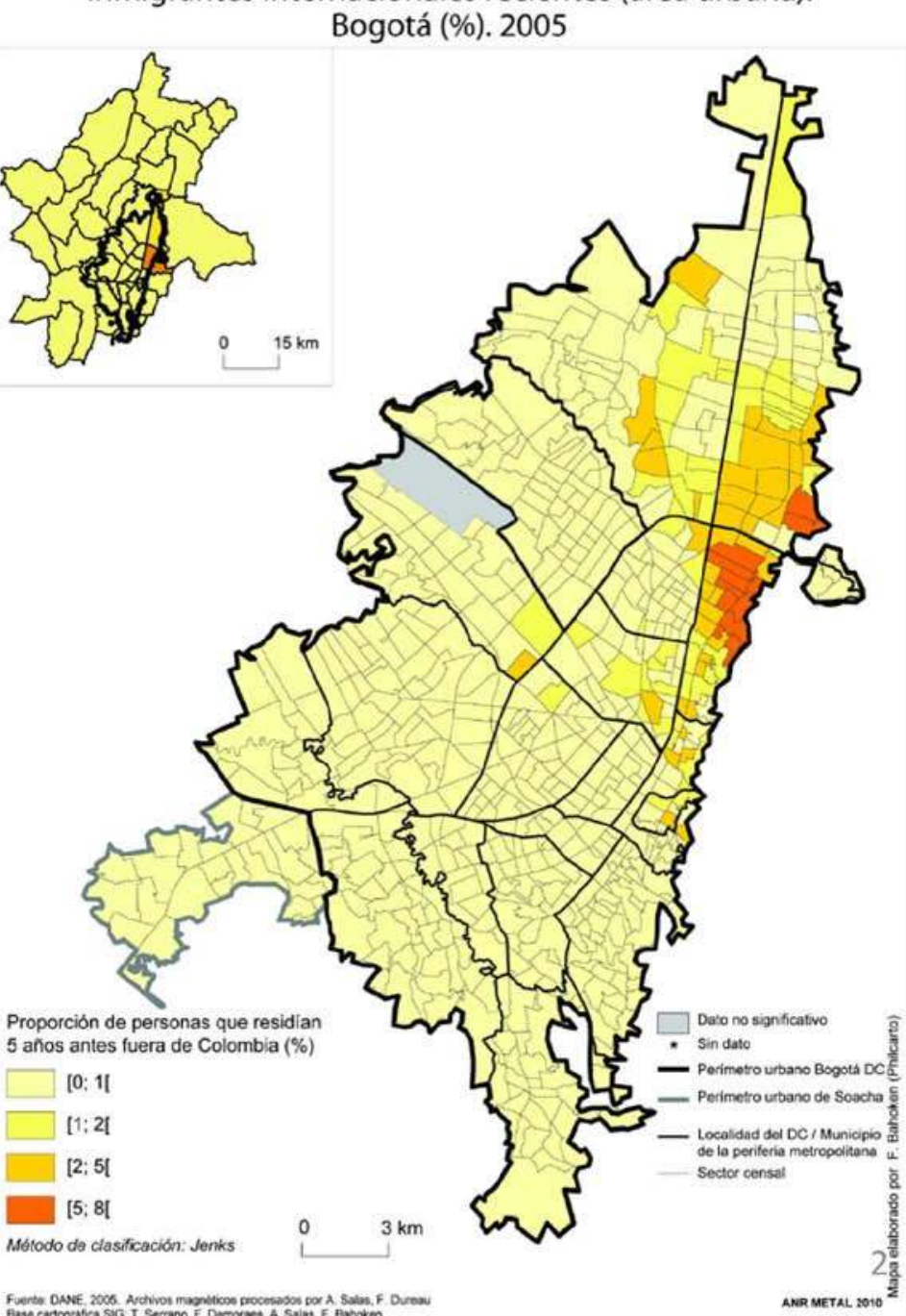


Figure $\mathbf{n}^{\circ} \mathbf{3}$ : Cartes des immigrants récents à Bogota selon le pays de résidence antérieur (2005).
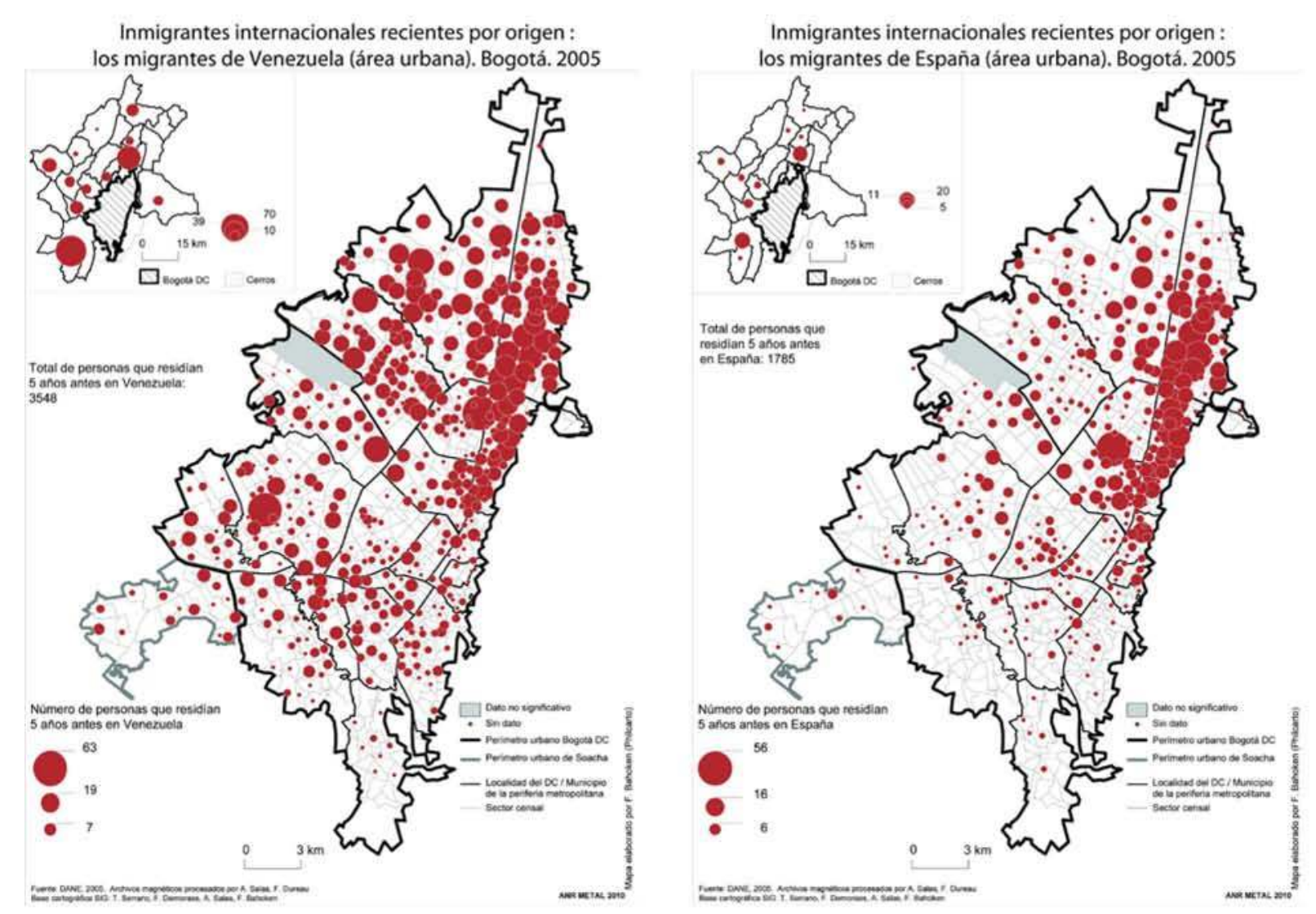

Inmigrantes internacionales recientes por origen : Inmigrantes internacionales recientes por origen :
los migrantes de Estados Unidos (área urbana). Bogotá. 2005

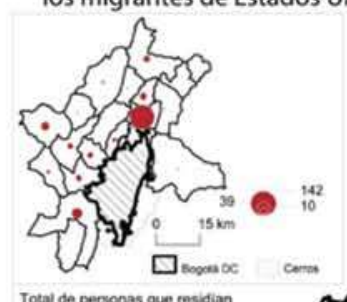
?दि (15) J) Total de perenonas que residian
5 ahos antes en Estadios Unidos.
7330

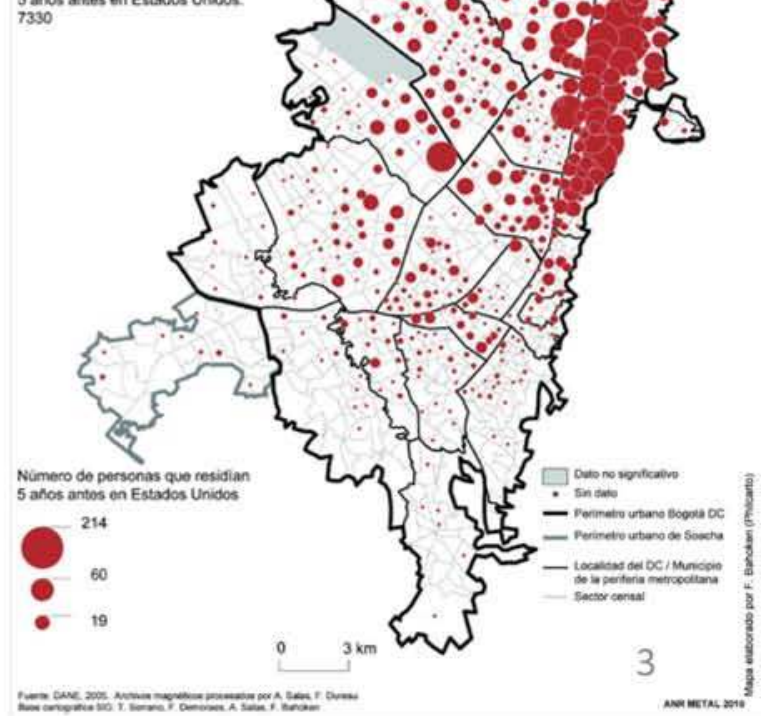
ร.

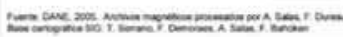


À partir des résultats du recensement colombien de 2005 , nous avons cartographié le nombre d'immigrants récents résidant en 2005 à Bogota, selon le pays de résidence en 2000 (Figure n³).

Attention: dans la carte des immigrants en provenance des États-Unis, les cercles ont des superficies qui ne suivent pas la règle de proportionnalité employée dans les deux autres cartes (migrants depuis le Venezuela et l'Espagne).

La migration en provenance du Venezuela, composée principalement de classes moyennes, correspond à deux flux : l'émigration depuis le Venezuela de jeunes adultes fuyant le régime d'Hugo Chavez (beaucoup d'entre eux sont des entrepreneurs) et la migration «de retour» de Colombiens ou de leurs enfants émigrés au Venezuela dans les années 1960-1970.

\section{Compter l'émigration dans les recensements latino-américains}

Nous avons vu que compter l'émigration constitue un enjeu important en Amérique latine. Une première solution correspond aux «méthodes directes»: le nombre d'émigrants est appréhendé à partir des immigrants comptabilisés dans les recensements des autres pays. Le projet Investifación de la Migración Internacional en Latino América (IMILA) (CELADE-UIESP) offre les résultats de l'exploitation des recensements depuis les années 1960 jusqu'aux plus récents. Sont disponibles les matrices origine/destination de migrants entre pays latino-américains, calculées pour cinq dates (la dernière correspond à 2006). Les méthodes indirectes reposent sur deux types de questions.

Le premier type de questions concerne les enfants vivants : il s'agit d'interroger les femmes de quinze ans et plus sur leurs enfants vivants qui résident à l'étranger. Ces questions sont généralement incluses dans le module du questionnaire portant sur la fécondité. Cette procédure employée dans certains recensements latino-américains des années 1980 (par exemple en 1985 en Uruguay), a généralement été abandonnée dans la vague des recensements des années 2000 (sauf en Haïti). Un avantage est de capter les émigrants sans critère d'appartenance à un ménage recensé dans le pays d'origine. Mais cette méthode présente aussi des limites importantes :

- ne sont captés que les enfants émigrés des femmes vivantes et n'ayant pas émigré ;

- on comptabilise des émigrants sur la durée de la vie, sans information sur la date ou le lieu de l'émigration.

Le second consiste à interroger les individus recensés sur les membres de leur ménage résidant à l'étranger : cette question a été introduite dans huit recensements réalisés au cours des années 2000 en Amérique latine (dont la Colombie en 2005). Cette méthode produit une sous-estimation des émigrants: ne sont pas comptés les émigrants appartenant à des ménages dont tous les membres ont émigré ou sont décédés, ou ceux dont le ménage a éclaté. On observe également une sous-déclaration des émigrés pour masquer leur migration illégale ou la réception de remises par le ménage enquêté. En outre, il est difficile d'identifier précisément qui faisait ou non partie du ménage, si le départ est ancien.

Le tableau, dressé par Alicia Maguid en 2008 (annexe $n^{\circ} 1$ ), précise la méthodologie employée dans les huit pays latino-américains qui ont inclus une mesure de l'émigration dans leurs recensements des années 2000. En dehors de la Colombie, tous sont localisés en Amérique centrale. Le Mexique est le seul pays qui a inclus une mesure des migrants de retour. Au Honduras, l'enjeu était d'évaluer les effets d'un désastre naturel (l'ouragan Mitch d'octobre 1998) en matière de migration internationale.

L'annexe $\mathrm{n}^{\circ} 2$ présente les recommandations du CELADE pour la 
vague des recensements des années 2010, présentées en annexe de la publication relative au recensement uruguayen de 2010 (Malacar, 2009). La figure $n^{\circ} 1$ (annexe $n^{\circ} 2$ ) montre les recommandations pour mesurer la migration interne et internationale. $\mathrm{La}$ figure $n^{\circ} 2$ (annexe $n^{\circ} 2$ ) précise les recommandations du CELADE pour la vague des recensements des années 2010, en ce qui concerne la mesure de l'émigration internationale.

L'annexe $n^{\circ} 3$ est un extrait du questionnaire du recensement colombien de 2005 qui présente les questions posées pour capter l'émigration internationale. Onze pays de destination sont proposés : y figurent les principaux pays de destination de l'émigration colombienne (Venezuela, ÉtatsUnis, Espagne) et six autres pays latinoaméricains, aux côtés de pays sensiblement moins importants pour l'émigration colombienne (comme le Canada et l'Australie).

Cette carte (Figure $\mathrm{n}^{\circ} 4$ ) relative à l'émigration récente est issue de l'exploitation des données recueillies à l'aide des questions présentées dans la figure précédente.

Par rapport aux cartes des immigrants, on observe une distribution spatiale bien différente. Celle-ci traduit le caractère diffus de l'émigration, qui touche des ménages résidant dans tous les types de quartier (du plus haut standing aux quartiers populaires), ce qui témoigne de la diversité sociale de l'émigration récente colombienne. Toutefois, on note que l'émigration récente ne touche pas les quartiers plus pauvres de la ville (à l'extrême sud-ouest). Il s'agit du phénomène classique de sélectivité de la migration (les plus pauvres ne disposent pas des ressources pour émigrer).

\section{La résidence en question}

Cette troisième partie évoque la question du rattachement des hommes aux lieux par la statistique. Dans la plupart des collectes démographiques, la résidence est définie comme le lieu où l'individu «a coutume d'habiter", le plus souvent considéré comme unique. C'est à travers ce filtre de la résidence unique et permanente que l'analyse de la mobilité spatiale a longtemps été limitée aux seules migrations correspondant à un transfert de résidence, laissant donc dans l'ombre toutes les autres formes de mobilités temporaires ou circulaires.

Le rattachement des hommes aux lieux par la statistique

«On appelle à proprement parler migration un ensemble de déplacements ayant pour effet de transférer la résidence des intéressés d'un certain lieu d'origine, ou lieu de départ, à un certain lieu de destination, ou lieu d'arrivée». Dictionnaire démographique multilingue.

- «On entend par résidence d'un individu, un lieu où il a coutume d'habiter». Dictionnaire démographique multilingue

Résidence : «Lieu où la personne a vécu la majeure partie des 12 mois précédant le recensement (sans inclure les absences temporaires pour vacances ou travail) ou, à défaut, où elle a l'intention de vivre au moins 6 mois ». Recommandations Nations Unies, 2008. 
Figure $\mathbf{n}^{\circ} 4$ : Cartes de l'émigration récente à Bogota (2005).
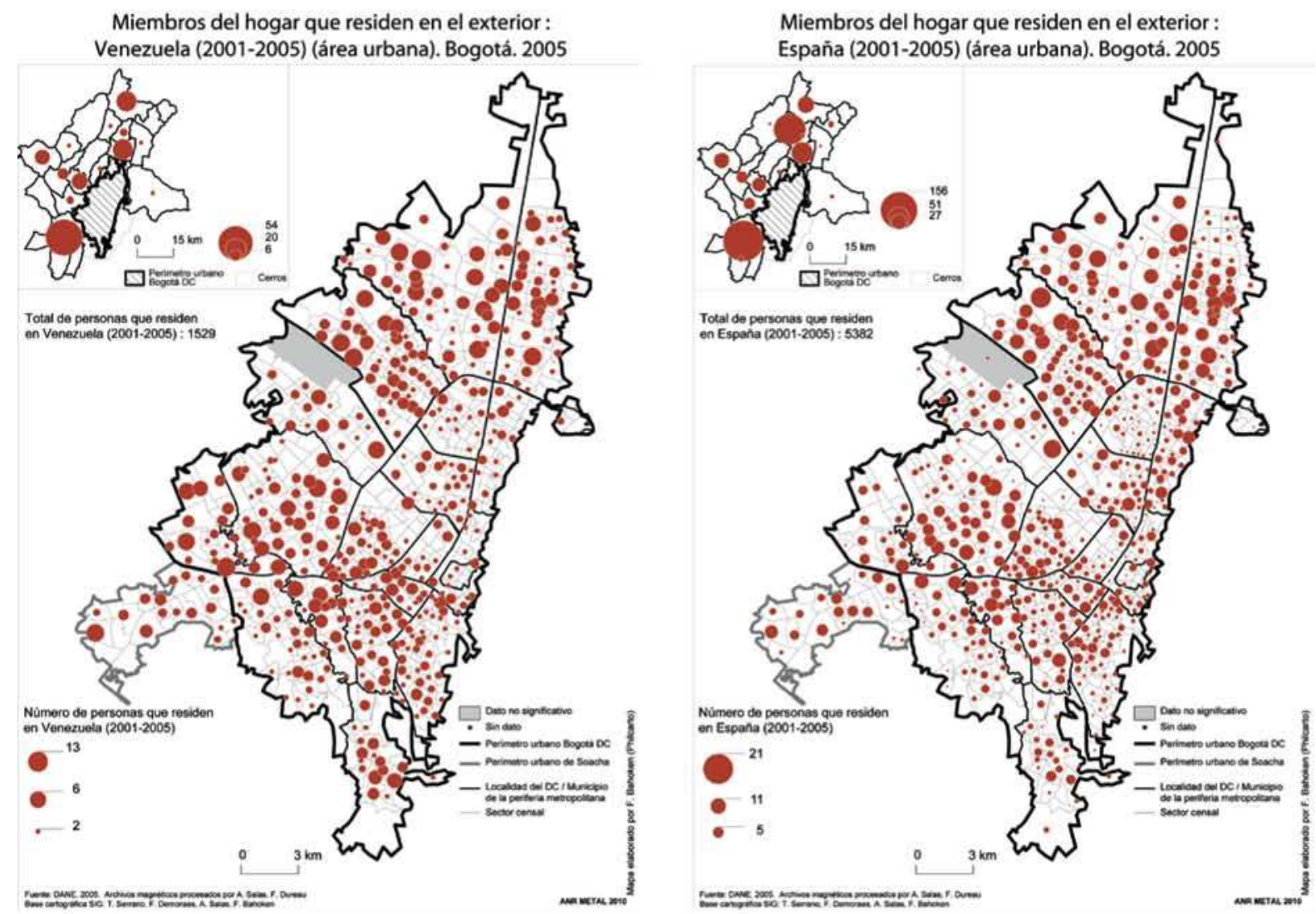

Miembros del hogar que residen en el exterior: Estados Unidos (2001-2005) (área urbana). Bogotá. 2005
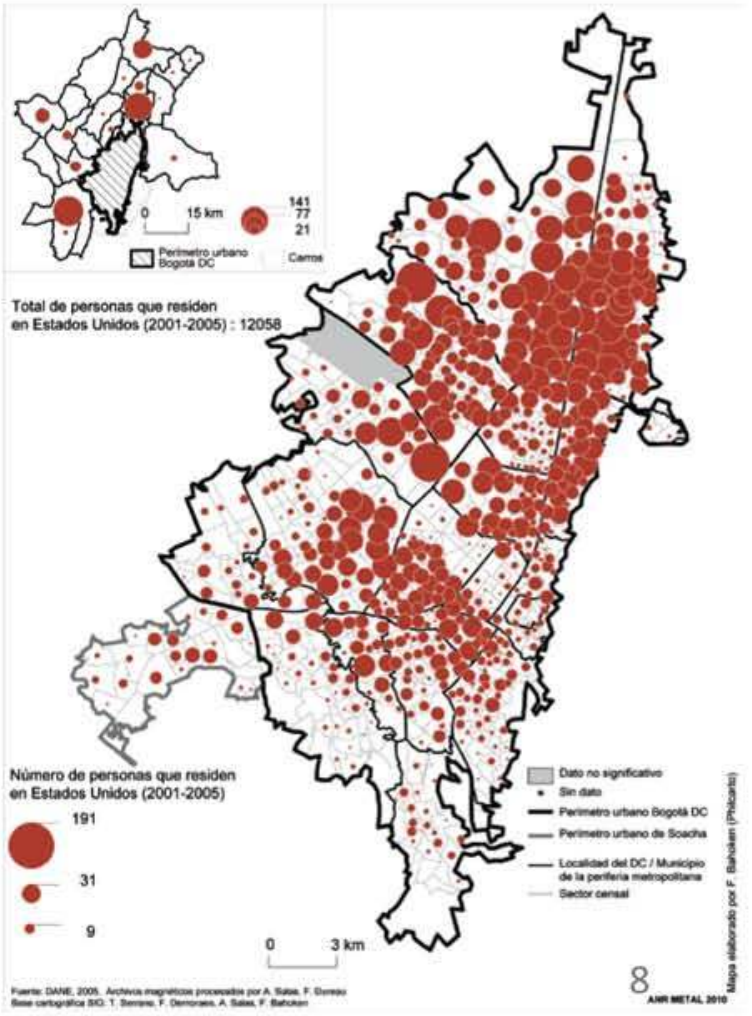
Les recommandations internationales en matière de statistique démographique continuent d'ailleurs d'identifier la multirésidence comme un problème, jamais comme un objet d'observation et de mesure. Les conséquences de cette appréhension réductrice du mode de rattachement des hommes aux lieux sont multiples. On peut évoquer, de façon très sommaire: la mauvaise appréhension des effectifs de population dans les contextes caractérisés par des fréquentations temporaires massives (les zones touristiques par exemple); le cas des villes comptant une proportion importante de population flottante ou de celles peuplées de populations « circulantes » alternant entre deux résidences géographiquement éloignées. De façon générale, la dynamique réelle de leur peuplement échappe à la comptabilité statistique, laissant de ce fait totalement démunis les organismes ayant en charge la gestion de ces territoires.

L'amélioration des transports s'est accompagnée d'une complexification des pratiques spatiales et des situations résidentielles. Ce dédoublement n'est pas un phénomène entièrement nouveau. JeanLoup Amselle évoquait dans les années 1970 «l'ubiquité des sociétés africaines» (Amselle et al., 1978) ; c'est aussi la pluri-localisation qui constituait (et constitue encore) la base de la reproduction des communautés paysannes dans les Andes. En Afrique comme en Amérique Latine, de nombreux travaux produits dans les trente ou quarante dernières années ont fait progresser, tant sur le plan des concepts que celui des méthodes, la saisie des situations résidentielles complexes. En France, c'est la bipolarité des pratiques résidentielles et des investissements des migrants internationaux qui a fait l'objet d'une reconnaissance précoce et partagée, que l'on retrouve par exemple sous le terme «d'espace de vie transnational » des migrants (Simon, 1995 ; Condon, 1996). L'analyse des populations immigrées en France a conduit à remettre en cause sans équivoque la notion de
« résidence principale », et à analyser au contraire les deux résidences dans leur complémentarité et leurs relations.

La complexité des pratiques résidentielles est réelle dans certains contextes ou dans certaines situations : migration internationale, processus de décohabitation, période de retraite, etc. Deux rencontres organisées en 2004, évoquées au début de cette intervention, ont permis d'avancer sur la question des pratiques pluri-résidentielles. Outre les résultats de travaux de recherche sur cette question, cela a donné l'occasion de voir comment la pluri-résidence était appréhendée par les systèmes nationaux de statistique, dans différentes régions du monde.

\section{Recensements de droit $v s$ recensements de fait}

La notion de résidence est à l'origine des recensements de droit. Dans une publication de 2008, Juan Chackiel (Chackiel, 2008) rappelle les avantages respectifs des deux types de recensement. Les avantages du recensement de fait (la population est dénombrée selon le lieu où elle est présente le jour du recensement) sont :

- la définition très simple de la population à dénombrer (alors que la population de droit dépend de l'application de critères subjectifs);

- les ménages totalement absents de leur résidence habituelle (cas plus fréquent s'il s'agit de ménages unipersonnels) sont comptabilisés (ce qui n'est pas le cas avec le recensement de droit);

- souvent réalisé avec immobilisation de la population : c'est bien une "photographie» instantanée de la population à un moment $\mathrm{T}$;

- les déplacements pour de courtes périodes sont saisis, à travers la comparaison entre le lieu de résidence au moment du recensement et le lieu de résidence habituel. 
Les avantages du recensement de droit (la population est dénombrée selon son lieu de résidence habituel) sont :

- le fait qu'il permette d'étaler la collecte dans le temps;

- et que les personnes résidentes habituelles qui sont à l'étranger au moment du recensement soient saisies, alors qu'elles ne le sont pas dans les recensements de fait (on ne connait la résidence habituelle que des présents dans le pays au moment du recensement).

Ces différents arguments conduisent Juan Chackiel à formuler une conclusion nuancée : «La décision est à prendre selon les circonstances particulières de chaque pays » (Chackiel, 2008: 34 ; traduction Françoise Dureau).

Il est toujours instructif de se pencher sur les instructions suivis par les agents recenseurs (annexe $\left.n^{\circ} 4\right)$ : c'est la seule façon de comprendre précisément comment sont produits les chiffres. De façon générale, on peut lire dans ces instructions aux enquêteurs établies pour le recensement uruguayen de 2011 le poids implicite de normes :

- les enfants de parents divorcés sont plutôt domiciliés chez la mère ;

- les travailleurs bi-résidents sont domiciliés à leur domicile familial alors que la majeure partie du temps ( 5 jours sur 7 ) ils vivent en dehors de ce logement. Dans le cas des travailleurs bi-résidents uruguayens, une bonne partie d'entre eux travaillent en Argentine: cela revient donc à nier la présence hors des frontières de ces individus qui travaillent et résident la majeure partie du temps à l'étranger.

La figure $n^{\circ} 2$ (annexe $n^{\circ} 4$ ) montre qu'une règle différente est appliquée pour les bi-résidents, selon que l'autre résidence est en Uruguay ou à l'étranger. Ce parti pris traduit vraisemblablement une volonté de «rapatrier» le maximum de population en Uruguay, comme on l'a vu pour les travailleurs bi-résidents.

\section{L'observation des systèmes résidentiels complexes}

Au-delà des recensements, il s'agit de voir maintenant comment nous pouvons envisager, dans le cadre d'enquêtes sur échantillon, d'avancer dans la connaissance des pratiques résidentielles complexes (Bonnin et de Villanova, 1999; Dureau, 2002 ; Pierre, 2006). L'enjeu est d'arriver à capter les systèmes de lieux pratiqués par les individus. Deux expériences d'enquête sont évoquées: l'une réalisée en 1993 à Bogota, l'autre en 1996 dans des villes pétrolières du Casanare, département situé à l'est de la Colombie. Les mêmes procédures de collecte ont été appliquées en 2009 dans les enquêtes METAL à Bogota, Santiago et São Paulo et dans l'enquête MEREV menée à Lisbonne. Enfin, nous évoquerons l'exemple de l'enquête Transport réalisée en France en 2007.

Pour décrire les situations résidentielles, deux notions sont souvent utilisées :

- celle de «système résidentiel familial»: cette notion n'est pas nouvelle. Nous l'avons reprise des travaux de l'équipe d'Émile Le Bris qui travaillait à la fin des années 1970 sur le rôle des transformations de l'habitat dans les transformations familiales dans des villes d'Afrique de l'Ouest. Cette équipe a proposé la notion de "système résidentiel familial », pour appréhender «un ensemble articulé de lieux de résidence (unités d'habitation) des membres d'une famille étendue ou élargie » (Le Bris et al., 1985). Cette notion est tout à fait voisine de celle de "ménage confédéré », proposée de l'autre côté de l'Atlantique par Jorge Balan et Jorge Dandler (1987) à propos des migrants boliviens, pour désigner «une unité composée de différents segments, qui seraient captés comme des ménages indépendants par les recensements; la localisation des segments obéit à une logique de reproduction multipolaire de la famille » - celle de «système de résidence»: nous l'utilisons pour désigner, au niveau de 
l'individu, la configuration spatio-temporelle définie par l'ensemble des logements habités par un individu au cours de l'année, l'intensité et le rythme de résidence dans chacun d'eux.

Dans les deux cas, pour les individus comme pour les familles, l'emploi du mot de « système» traduit une volonté d'insister sur l'articulation entre les lieux et sur les pratiques les mettant en relation.

Pour les systèmes de résidence des individus, l'élément fondamental du recueil d'informations réside dans la présentation dans un calendrier des séjours dans les différents logements au cours des douze mois précédant l'enquête (annexe $n^{\circ} 5$ ). Pour chaque personne, en plus du logement où est réalisée l'enquête, sont identifiés deux autres logements. La durée minimale de séjour dans un logement pour que celui-ci soit pris en compte en tant qu' «autre logement », c'est-à-dire autre composante du système de résidence, est de 28/30 jours, consécutifs ou non, au cours de l'année précédant l'enquête.

Une phase d'apprentissage du maniement du calendrier par les enquêteurs a bien sûr été nécessaire. Mais, une fois cet apprentissage effectué, l'application de cette section du questionnaire n'a pas posé de problèmes. La solution adoptée s'est révélée efficace: la représentation graphique des séjours dans les différents logements a permis de transcrire aisément des situations complexes. Le graphique a joué un rôle important pour faire assimiler correctement par les enquêteurs le concept de «système de résidence », en tant que combinaison de plusieurs lieux de séjour de la personne interrogée. Les deux expériences colombiennes, et leur réplique en 1995 à Delhi par Véronique Dupont, montrent l'efficacité des procédures graphiques pour capter les situations irrégulières, instables ou intermittentes vécues par certaines personnes
Dans l'enquête réalisée à Bogota en 2009 (annexe $\mathrm{n}^{\circ}$ ) $), 18 \%$ des individus ont déclaré un autre logement où ils ont résidé plus de 30 jours consécutifs ou non au cours de l'année écoulée, et $1 \%$ deux autres logements fréquentés plus de 30 jours.

La procédure de codage a été sensiblement améliorée dans l'enquête METAL par rapport aux collectes antérieures: il a ainsi été possible de conserver la richesse de l'information transcrite graphiquement dans le calendrier.

Pour chacun des logements identifiés, on dispose donc de l'information suivante: - le nombre de jours passés dans le logement au cours des douze mois précédant l'enquête ;

- le rythme de fréquentation de ce logement (continuité / alternance);

- la localisation précise du logement;

- la nature (logement d'Ego, logement d'un parent, hôtel, internat, centre d'exploitation pétrolière, etc.) ;

- la raison du séjour dans ce logement (travail, étude, famille, etc.).

Dans cette section comme dans l'ensemble du questionnaire, les lieux ne sont pas précodés et font l'objet d'une description précise en clair (adresse ou quartier dans la ville d'enquête, Bogota ou villes pétrolières du Casanare, commune en dehors).

Un certain nombre de variables synthétiques sont créées à partir des informations du questionnaire.

À partir de l'information sur le nombre de jours qui peut concerner jusqu'à trois logements, on calcule une «densité de résidence » dans chaque logement (rapport du nombre de jours passés dans le logement et du total de jours d'observation).

La combinaison des informations relatives au nombre de logements où la personne a séjourné plus de $28 / 30$ jours, et aux caractéristiques temporelles de (des) 
séjour(s) dans chaque logement permet de construire pour chacun des individus observés une variable "système de résidence». Cette variable synthétique combine les modalités de base suivantes :

- système unipolaire (un seul lieu de résidence),

- système bipolaire (alternance résidentielle entre deux logements dans lesquels la personne réside par périodes) avec différentes catégories selon les densités de résidence dans les logements,

- système itinérant (succession de logements, avec des déménagements successifs), etc.

On peut qualifier ce système de résidence selon la localisation des différents logements : système bipolaire international/national, système itinérant international/national, etc.

\section{Les systèmes résidentiels bipolaires dans les villes du Casanare}

Dans l'enquête réalisée en 1996 dans le Casanare, on observe ainsi des systèmes résidentiels bipolaires associés à l'éclatement géographique des familles (pour les Casanareños comme pour les immigrants récents attirés par l'économie liée à la production pétrolière).

La circulation entre deux résidences est une pratique relativement fréquente : sur l'ensemble de la population, 9\% des individus ont un système bipolaire, $8 \%$ un système itinérant, et $83 \%$ un système unipolaire. Dans deux des villes étudiées (Yopal et Aguazul), la circulation est deux fois plus fréquente chez les hommes que chez les femmes. La circulation est une pratique «au long cours»: un tiers des migrants bipolaires de Yopal ont plus de cinq ans d'ancienneté dans la ville, $20 \%$ sont même arrivés il y a plus de dix ans. L'éloignement spatial est parfois considérable. Les séjours dans l'autre résidence sont réalisés à des rythmes variés (un quart selon un rythme hebdomadaire, un quart selon un rythme mensuel), leur fréquence n'étant pas directement liée à la distance: on observe une grande variété pour une même localisation de l'autre résidence. Ainsi, les travailleurs du pétrole ont une circulation particulièrement intense, dont les rythmes sont étroitement liés aux revenus (fréquence régulière et soutenue pour les ingénieurs; fréquence moindre et plus aléatoire pour les ouvriers non qualifiés).

Différents modèles de systèmes résidentiels familiaux ont été identifiés, associés à des rythmes de circulation variables :

- ingénieurs ayant leur famille à Bogota, venant travailler chaque semaine dans le Casanare ;

- ouvriers qualifiés du pétrole: leur famille réside ailleurs dans le pays, eux suivent l'activité pétrolière ;

- agriculteurs du Casanare ayant leur famille à Yopal et faisant des allers-retours entre Yopal et leur ferme (ce qui est à mettre en lien avec le problème de scolarisation dans les campagnes et le conflit armé) ;

- avec l'aggravation du conflit, les familles sont envoyées encore plus loin, dans le Boyaca ou à Bogota.

\section{La circulation entre métropoles européennes}

Dans le projet MEREV, les enquêtes se sont déroulées en trois phases :

1) application d'un questionnaire court dans des lieux de transport (aéroport, gare ferroviaire, gare routière) ;

2) application d'un questionnaire biographique à un sous-échantillon de la phase 1 ;

3) réalisation d'entretiens à un souséchantillon de la phase 2.

Les résultats issus de ce projet (Imbert et al., 2013) mettent en évidence des pratiques résidentielles complexes à dimension internationale, comme l'exemple présenté dans l'annexe $n^{\circ} 7$. 


\section{L'enquête nationale sur les transports et les déplacements (2007-2008)}

Le rapport d'Armoogun et al. (2007) est une excellente synthèse des méthodes employées pour les «Enquêtes Nationales Transport» dans 17 pays ayant une expérience dans ce domaine. L'enquête «Nationale Transports et déplacements » (ENTD) réalisée en France en 2007-2008 présente des innovations méthodologiques importantes par rapport aux enquêtes précédentes (1967, 1974, 1982, 1994). Ses principales caractéristiques sont :

- un échantillon de 20000 ménages (environ 50000 individus);

- un questionnaire administré par un enquêteur, au cours de deux visites ;

- un questionnaire descriptif du logement, du ménage et de l'ensemble de ses membres ;

- un carnet de véhicule renseigné par l'un des conducteurs pendant sept jours ;

- une interrogation d'un individu par ménage âgé de six ans ou plus sur ses propres déplacements de la veille, du dernier week-end, de la semaine, de la mobilité à longue distance sur trois mois.

Sont mesurées la mobilité locale (la veille et le dernier samedi ou dimanche) et la mobilité à longue distance (sur trois mois). En 2007, un suivi des déplacements avec des récepteurs GPS confiés à 750 individus volontaires a été testé et un module biographique a été appliqué à 1000 individus. La mobilité résidentielle était la grande absente des «Enquêtes Nationales Transports» et des «Enquêtes Ménages Déplacements», en France comme à l'étranger. Seule l'ENTD de 2007 échappe partiellement à ce constat: le module biographique administré à titre expérimental à un sous-échantillon permet de saisir l'évolution des pratiques de déplacement au cours de la vie, et de la mettre en relation avec les trajectoires résidentielle et professionnelle des individus. Cette enquête présente aussi des solutions intéressantes pour sortir de la vision de la résidence unique et capter des pratiques résidentielles complexes.

Un des intérêts du questionnaire de l'ENTD 2007 (annexe $n^{\circ} 8$ ), outre la saisie des déplacements à longue distance sur trois mois, réside dans la saisie de la multirésidence, grâce au module « Occupation du logement ». Sont identifiées les personnes qui ne vivent pas de façon continue dans le logement enquêté. Et, pour chacune d'elles, sont saisies cinq catégories de fréquence de présence dans le logement. Pour chaque catégorie, une question vise à cerner le nombre de jours de présence dans le logement (ce qui permet de calculer une "densité de résidence» dans le logement). Sont également appréhendés: le type de l'autre logement fréquenté s'il s'agit d'un logement collectif et le nombre d'autres logements individuels fréquentés.

Chacun des autres logements individuels fréquentés par ces individus est caractérisé par une série de questions présentées dans la figure $\mathrm{n}^{\circ} 2$ de l'annexe $\mathrm{n}^{\circ} 8$ :

- localisation (France/étranger);

- individus qui vivent dans le logement enquêté et fréquentent cet autre logement ; - autres individus qui vivent dans l'autre logement.

Une question porte sur le partage des dépenses, ce qui renvoie au repérage des unités domestiques selon un critère budgétaire (Figure $n^{\circ} 3$, annexe $n^{\circ} 8$ ). Cette approche correspond à celle mise en œuvre pour l'identification des ménages dans de nombreux pays latino-américains : au critère de corésidence dans un même logement, s'articule un critère de partage des frais de nourriture. 


\section{Conclusion}

Bibliographie

Les exemples déclinés dans cette présentation l'ont montré, l'expérience construite au Sud constitue un apport appréciable pour appréhender des situations complexes longtemps niées par la statistique pensée au Nord.

Il faut aussi souligner l'apport d'une vision globale des pratiques de mobilité, pour appréhender les pratiques complexes échappant au système traditionnel de production statistique sur les mobilités: l'enjeu actuel est bien de capter ce qui ne relève, ni de la mobilité locale dite quotidienne, ni de la migration en tant que transfert d'une résidence supposée unique et permanente.

Il existe de nombreuses façons d'avancer dans la connaissance de la migration internationale, en allant voir ce qui se produit comme information ailleurs et/ou dans d'autres champs d'étude de la mobilité.

Françoise Dureau DR IRD honoraire Migrinter - UMR 7301 f.dureau@gmail.com
Aghassian, Michel ; Amselle, Jean-Loup ; Saliou-Baldé, Mamadou (1978) Les migrations africaines. Réseaux et processus migratoires, Paris, Maspero, 127 p.

Armoogun, Jimmy ; Hubert, Jean-Paul ; Bonnel, Patrick; Madre, Jean-Loup (2007) Préparer la prochaine enquête nationale transport avec un regard international, Rapport de convention 03MT68 pour la DRAST, 110 p.

Balán, Jorge ; Dandler, Jorge (1987) Marriage process and household formation: migration in the Cochabamba region (Bolivia) and Bolivian migrants in Buenos Aires (Argentina), Communication présentée au séminaire sur l'insertion des migrants dans les villes africaines, CRDIORSTOM-URD, Lomé, 10-14 février 1987, $47 \mathrm{p}$.

Bonnin, Philippe; Villanova, Roselyne de (1999) D'une maison l'autre. Parcours et mobilités résidentielles, Paris, Creaphis, $371 \mathrm{p}$.

Chackiel, Juan (2008) Censo, residencia habitual y movilidad territorial, Notas de población, n 88 , pp. 21-61.

Condon, Stéphanie (1996) Les migrants antillais en métropole: un espace de vie transatlantique, L'espace géographique, $\mathrm{n}^{\circ} 2-3$, pp. 513-520.

Dureau, Françoise (2002) Les systèmes résidentiels : concepts et applications, in Lévy, J.-P. ; Dureau, F. (dir.) L'accès à la ville. Les mobilités spatiales en question, Paris, L'Harmattan, pp. 355-382 (Habitat et sociétés).

Dureau, Françoise (2006) L'observation des systèmes résidentiels dans les villes colombiennes, in Dureau, F.; Golaz, V. (dir.) Mobilité et résidence, Paris, CEPED, 15 p. (Les Numériques du Ceped) Disponible sur Internet] 
Dureau, Françoise ; Golaz, Valérie (2006) Mobilité et résidence. Actes de l'atelier international, CEPED, 16-17 novembre 2004, Nogent-surMarne, Paris, CEPED, (Les numériques du Ceped) Disponible sur Internet]

Henry, Louis (1981) Dictionnaire démographique multilingue, volume français, Liège, UIESPOrdina éditions, 179 p.

Imbert, Christophe (dir.) ; Dubucs Hadrien ; Dureau, Françoise ; Giroud, Matthieu (2014) D'une métropole à l'autre: pratiques urbaines et circulations dans l'espace européen, Paris, Armand Colin 484 p. (Recherches).

Le Bris, Émile; Marie, Alain; Osmont, Annick; Sinou, Alain (1985) Résidence, stratégies, parenté dans les villes africaines, Les annales de la recherche urbaine, $\mathrm{n}^{\circ} 25$, pp. 1330.

Maguid, Alicia Mirta (2008) La emigración internacional a través de los censos en países de origen: evaluación de resultados y recomendaciones, (, Santiago de Chile, CEPAL, LC/L.2968-P (Población y Desarrollo, $n^{\circ} 86$ ).

Malacar, Daniel (2009) El relevamiento de la migracion interna e interncional en el censo de Uruguay 2010, Montevideo, INE - OIM, 84 p.

Naciones Unidas (2008) Principles and Recommendations for Population and Housing Censuses. Revision 2, New-York, Éd. Naciones Unidas, ST/ESA/STAT/SER.M/67/Rev.2, (Series M, no 67/Rev.2).

Papon, Francis ; Marchal, Marina; Roux, Sophie; Marchal, Philippe ; Armoogun, Jimmy (2010) Parcours individuels et histoire de la mobilité. Analyse du volet "biographie" de l'Enquête Nationale sur les Transports et les Déplacements 2007-2008, Paris, Inrets, Département Économie et Sociologie des Transports, $204 \mathrm{p}$.
Pierre, Magali (2006) L'invention d'un système bi-résidentiel. Un choix de vie pour couple retraité aisé, Annales de la recherche urbaine, n¹00, pp. 107-113.

Simon, Gildas (1995) Géodynamique des migrations internationales dans le monde, Paris, PUF, $429 \mathrm{p}$. 
Annexe $\mathbf{n}^{\circ} \mathbf{1}$ : Tableau décrivant la méthodologie employée dans les huit pays latino-américains qui ont inclus une mesure de l'émigration dans leurs recensements des années 2000.

\begin{tabular}{|c|c|c|c|}
\hline \multicolumn{4}{|c|}{$\begin{array}{l}\text { AMÉRICA LATINA: MODO DE DEFINICIÓN DE LOS EMIGRANTES } \\
\text { EN LOS PAISES SELECCIONADOS, RONDA CENSAL DE } 2000\end{array}$} \\
\hline $\begin{array}{l}\text { País y fecha } \\
\text { del censo }\end{array}$ & Pregunta & Universo de emigrantes & \multirow{9}{*}{ 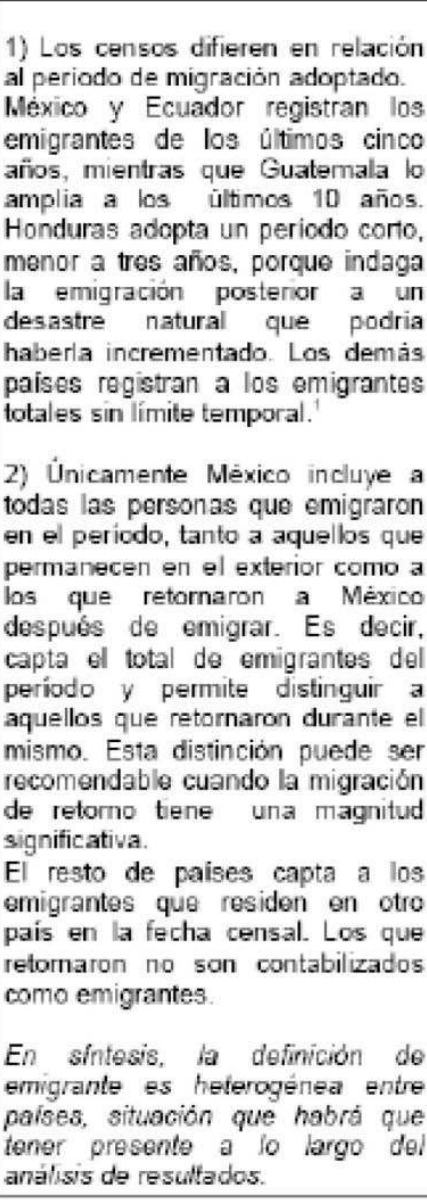 } \\
\hline $\begin{array}{l}\text { México } \\
\text { Febrero } 2000\end{array}$ & $\begin{array}{l}\text { ¿Durante los últimos } 5 \text { años, } \\
\text { desde enero de } 1995 \text { a la facha, } \\
\text { alguna persona que vive o vivia } \\
\text { en este hogar se fue a vivir a } \\
\text { otro pais? }\end{array}$ & $\begin{array}{l}\text { Emigrantes de periodo, } \\
\text { incluyendo a los que } \\
\text { retornaron } \\
\text { Período: } 1995-2000\end{array}$ & \\
\hline $\begin{array}{l}\text { Guatemala } \\
2002\end{array}$ & $\begin{array}{l}\text { ¿En los últimos } 10 \text { años, alguna } \\
\text { persona de este hogar se fue a } \\
\text { vivir permanentemente a otro } \\
\text { país? }\end{array}$ & $\begin{array}{l}\text { Emigrantes de periodo } \\
\text { que no retornaron } \\
\text { Período: } 1992-2002\end{array}$ & \\
\hline $\begin{array}{l}\text { Ecuador } \\
\text { Noviembre } 2001\end{array}$ & $\begin{array}{l}\text { ¿A partir de noviembre de } 1996 \\
\text { (durante los últimos } 5 \text { afios), una } \\
\text { o mas personas que fueron } \\
\text { miembros de este hogar viajaron } \\
\text { a otro pais y todavia no } \\
\text { retornan? }\end{array}$ & $\begin{array}{l}\text { Emigrantes de periodo } \\
\text { que no retornaron } \\
\text { Período: } 1996-2001\end{array}$ & \\
\hline $\begin{array}{l}\text { República } \\
\text { Dominicana } \\
\text { Octubre } 2002 \\
\end{array}$ & $\begin{array}{l}\text { ¿Alguna de las personas que } \\
\text { residian en este hogar vive fuera } \\
\text { del pais? }\end{array}$ & $\begin{array}{l}\text { Emigrantes totales que } \\
\text { no retornaron } \\
\text { (emigración absoluta) }\end{array}$ & \\
\hline $\begin{array}{l}\text { Nicaragua } \\
\text { Marzo-junio } \\
2005\end{array}$ & $\begin{array}{l}\text { Alguna persona que era } \\
\text { miembro de este hogar, ¿vive } \\
\text { actualmente en otro pais? }\end{array}$ & $\begin{array}{l}\text { Emigrantes totales que } \\
\text { no retornaron } \\
\text { (emigración absoluta) }\end{array}$ & \\
\hline $\begin{array}{l}\text { Colombia } \\
\text { Mayo } 2005\end{array}$ & $\begin{array}{l}\text { ¿Alguna o algunas personas, } \\
\text { siendo miembros de este hogar, } \\
\text { se ha ido a vivir de MANERA } \\
\text { PERMANENTE al exterior? }\end{array}$ & $\begin{array}{l}\text { Emigrantes totales que } \\
\text { no retornaron } \\
\text { (emigración absoluta) }\end{array}$ & \\
\hline $\begin{array}{l}\text { El Salvador } \\
\text { Mayo } 2007\end{array}$ & $\begin{array}{l}\text { Alguna persona miembro de este } \\
\text { hogar. ¿se fue a vivir } \\
\text { permanentemente a otro pais? }\end{array}$ & $\begin{array}{l}\text { Emigrantes totales que } \\
\text { no retornaron } \\
\text { (emigración absoluta) }\end{array}$ & \\
\hline $\begin{array}{l}\text { Honduras } \\
\text { Julio } 2001\end{array}$ & $\begin{array}{l}\text { Después del Huracán Mitch } \\
\text { (cctubre 1998), calguna persona } \\
\text { que pertenecia a este hogar se } \\
\text { ha ido a vivir a otro pais? }\end{array}$ & & \\
\hline
\end{tabular}

Fuente: Maguid 2008 
Annexe n² : Recommandations CELADE.

Figure $\mathbf{n}^{\circ} 1$.

\section{PREGUNTAS PARA CAPTAR MIGRACION INTERNA E INTERNACIONAL}

EN MODULO DE LAS PERSONAS DEL HOGAR

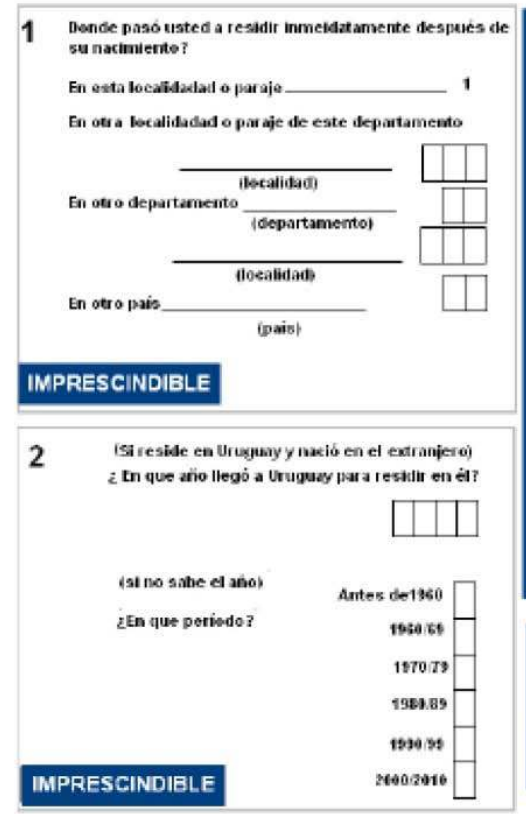

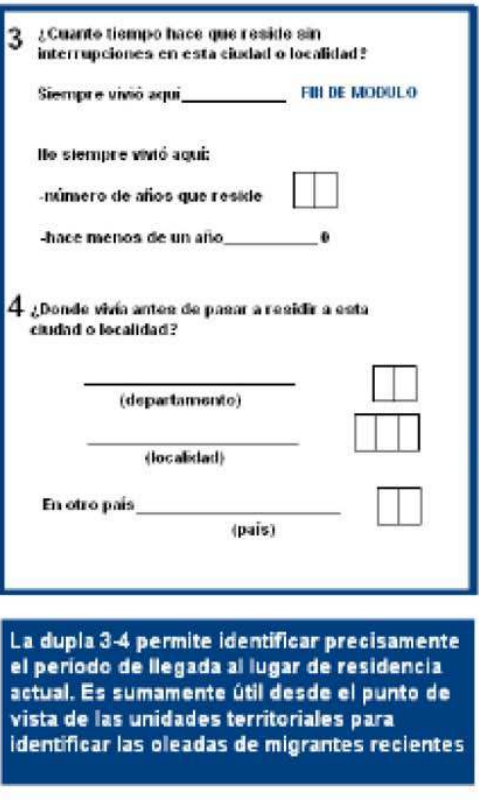

PARA PERSGGLAS RE 2 AIIOS $Y$ MAS

5 ¿00acle wivia hace 005 años (en 26ta)?

Its habia nacido _ 1 FIII DE MOVULO

En esta loctalihadad o paraice__ 2

Eh our locificlatial o paraje de este separtamento

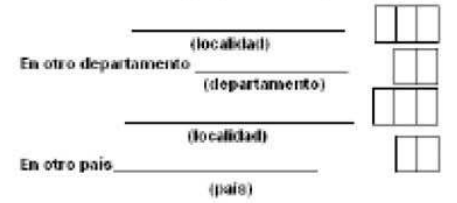

NUEVA SUOERERTCIA CELADE PAFA CAPTAR MICRACION MAS RECIENTE

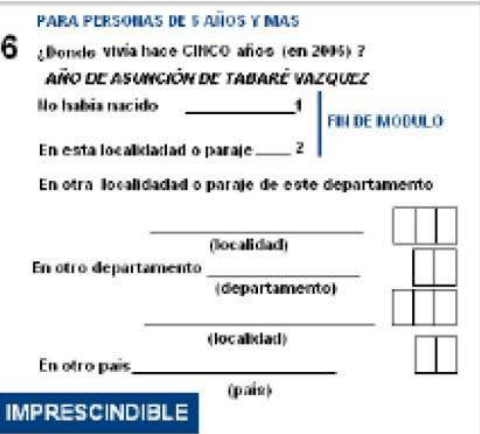

PARA PLFSOHAS DE 5 AIIOS $Y$ MAS Ho babia sacido

En otra locallidadud o parate de oste olepartamento vista de las unidades territoriales para dentificar las oleadas de migrantes recientes

Figure $n^{\circ} 2$.

\section{PREGUNTAS PARA CAPTAR EMIGRACION INTERNACIONAL}

PARA CADA MIRANTE (DE 1A N)

\section{LUEGO DE MODULO DE HOGAR}

\section{EMIORACION INTERNACIONAL}

1. ¿Dutante los tiltimos 10 años alyuna porsonaque vivia con ustedes se ftué a vivir a otro pais y mo ha regresado a vivir a Uruguay? (desde el lero de enero de 2000)

$$
\stackrel{\text { NI_ }}{2} \text { ? FIN DE MODULO }
$$

$2 \cdot 2$ Cuantas personas?

$-N$

Digame el nombre de pita de todas las personas

(registre los nombres en el encabezamiento de cada una de las personas)

\section{EMIGRANTE: Nombre}

3. Cuando se fue

St ¿vivia con ustedes?

NO 2 PASE AL SIGUIENTE EMIGRANTE

A. ¿Es varon o mujer?

Varón__

5- ¿Cual os la rolación de parentozco con ol jefo o jefa de este hogan?

\begin{tabular}{|c|c|c|}
\hline \multicolumn{3}{|c|}{ conyuge/pareja } \\
\hline \\
\hline \multicolumn{3}{|c|}{$\begin{array}{l}\text { yerno/nuera } \\
\text { nieto/a }\end{array}$} \\
\hline \multicolumn{3}{|l|}{ padres'suegros } \\
\hline \multicolumn{3}{|l|}{ otro pariente } \\
\hline \multicolumn{2}{|l|}{ otro no pariento } & \\
\hline nque ano se fue? & $20-\ldots$ & \\
\hline \multicolumn{2}{|c|}{ que odad tonia cumado se fue? } & -- \\
\hline \multicolumn{2}{|c|}{ En que departamento vivia cuando se fue? } & - - \\
\hline \multicolumn{2}{|c|}{ en que pais reside actuaimente? } & - - \\
\hline
\end{tabular}


Annexe $\mathbf{n}^{\circ} \mathbf{3}$ : Extrait du questionnaire du recensement colombien de 2005.

22. ¿Alguna o algunas personas, siendo miembros de este hogar, se han ido a vivir de MANERA PERMANENTE al exterior? (BÁSICO)

1. $\mathrm{S}$

2. No

(pase a CTL3)

1.1 ¿Cuántas en total?

1.2 ¿En qué países residen actualmente y en cuál de los siguientes periodos se produjo su salida?

País de residencia actual

Venezuela

Estados Unidos

España

México

Costa Rica

Canadá

Australia

Ecuador

Panamá

Perú

Bolivia

Otro país
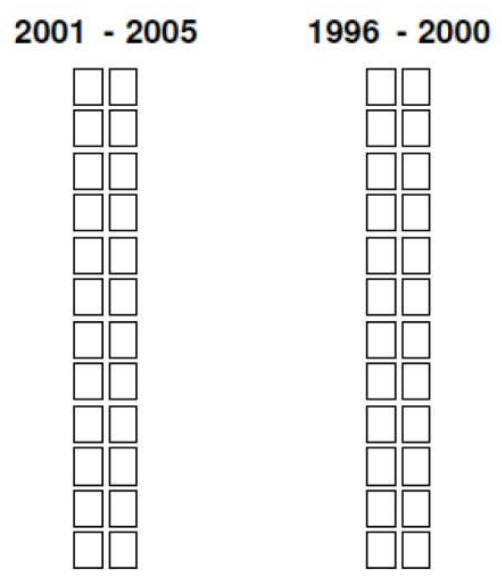

Antes de 1996

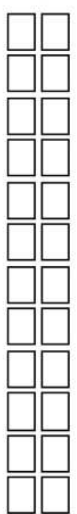


Annexe $\mathbf{n}^{\circ} \mathbf{4}$ : Instructions aux agents recenseurs, recensement Uruguay 2011.

Figure $\mathbf{n}^{\circ} 1$.

Residente Habitual es la persona que ha vivido en la vivienda la mayor parte de los últimos doce meses o que ha vivido menos tiempo pero se propone seguir viviendo alli.

\begin{abstract}
Casos especiales
Estudiantes de nivel terciario que están estudiando lejos de su hogar familiar.

Estudiantes de primaria o secundaria que están estudiando lejos de su hogar familiar.

Niños y adolescentes con doble residencia (hijos de padres separados o divorciados).

Trabajadores con doble residencia (personas que pasan la semana laboral en una residencia cercana a su trabajo y los fines de semana en su hogar familiar) o trabajadores cuya tarea implica un cambio constante de localidades de estadía (vendedores, consultores, conductores de medios de transporte de largo alcance, trabajadores de barcos pesqueros en alta mar, trabajadores zafrales en lugares distantes de su residencia familiar).
\end{abstract}

\section{Lugar donde debe ser censado \\ En la vivienda donde pernoctan la mayor parte de la semana.}

En la vivienda del hogar familiar.

En la vivienda donde pernocten la mayor parte de la semana (en caso de empate se define por la residencia de la madre o familiar en primera línea de consanguinidad).

En la vivienda del hogar familiar.

Excepción: el trabajador que vive solo es censado en el lugar donde se le encuentre. Se le debe aclarar que no debe ser censado nuevamente en su otra residencia. De todas maneras registre en observaciones la dirección (incluyendo localidad y departamento) de su otra residencia para permitir un control posterior.

Figure $n^{\circ} 2$.

Personas que tienen dos o más residencias habituales en donde pasan la misma cantidad de tiempo.
En caso de empate entre una residencia en Uruguay y otra en otro país, se define como residencia habitual la residencia de Uruguay (sean nacionales o extranjeros).

En caso de empate entre dos residencias en Uruguay se solicita al informante que se defina por una. Se le aclara que no debe ser censado en su otra residencia. 
Annexe n5 : La technique de collecte (Bogota 1993, Casanare 1996).

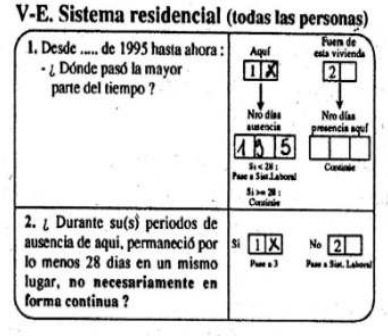

V.F. Sistema laboral (personas de 5 ańos o más) Paral los que no trabajaban la semana anterior:

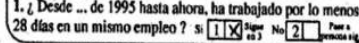

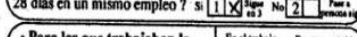
- Para los que trabajaban la semana anterior (Cap. V.C,
102 en ocup. princ. o sec) 1. Desde .... de 1995 hasta aho alor: la mayor parte del tiempo ? 2. 2 Durante su(s) periodos fuera
del trabajo principal actual, permanecio prom bo mal actual, en un mismo trabajo, no necesse-
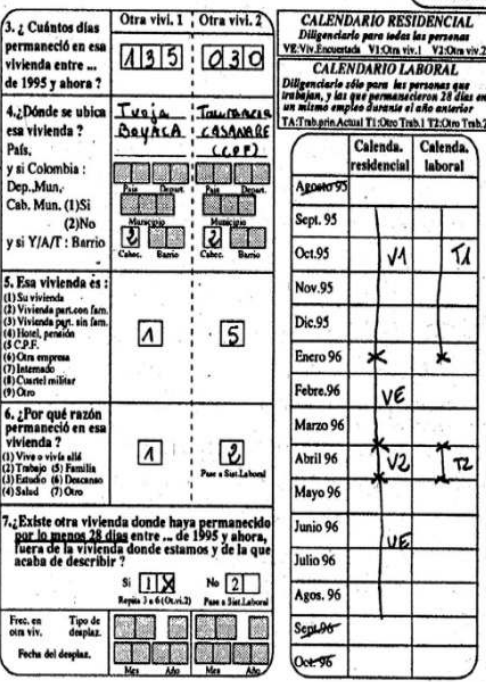

.11.

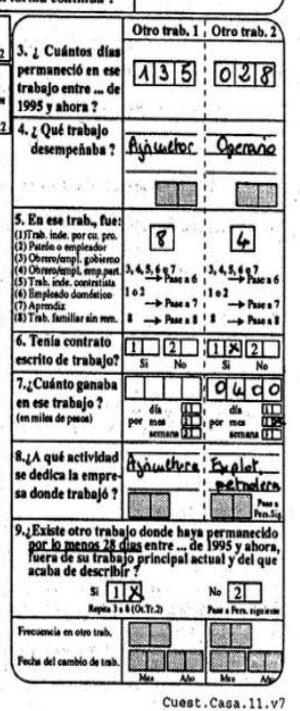

Formation enquêteurs

Rôle du calendrier pour transcrire des situations complexes

\section{Villes pétrolières : utilisation calendrier par jour pour fréquence de prestation des services publics}

\section{Autre application : Enquête Delhi, 1995}

Annexe $\mathbf{n}^{\circ} \mathbf{6}$ : La collecte des systèmes résidentiels (METAL, 2009).

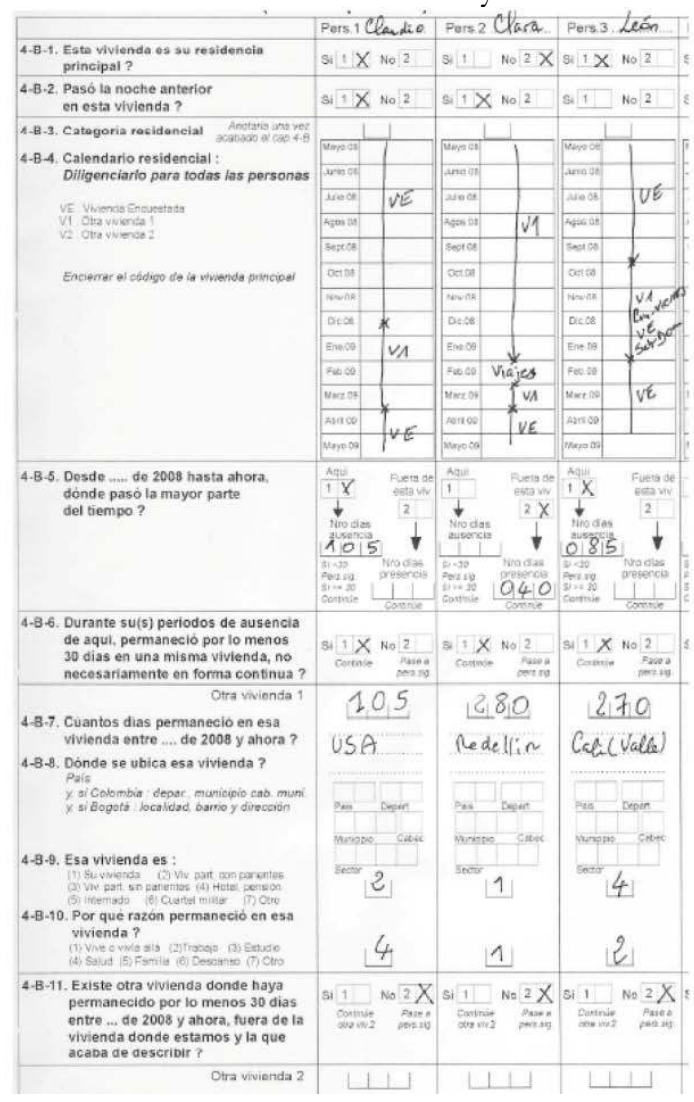

Persona 1: La persona estuvo viviendo en Miami, por razón de salud, en la casa de su hermana, de la mitad de diciembre de 2008 hasta finales de marzo de 2009.

Persona 2: La persona vive la mayoría del tiempo fuera de la vivienda encuestada, donde se quedó únicamente desde el principio del mes de abril de 2009 hasta la fecha de la encuesta, y el resto del año se quedo en su vivienda en Medellín (excepto el mes de Febrero, durante el cual viajó a muchos sitios).

Persona 3: La persona, de mayo de 2008 a finales de septiembre de 2008 vivió únicamente en la vivienda de la entrevista. Desde octubre de 2008 hasta finales de enero de 2009, por razón de trabajo, vive de Lunes a Viernes en Cali, y en la vivienda de la entrevista, en Bogotá, cada fin de semana. Desde febrero, se quedó todo el tiempo en su vivienda de Bogotá. 


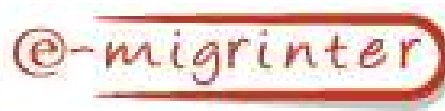

Annexe $\mathbf{n}^{\circ} 7$ : Trajectoire résidentielle de Sergio (enquête MEREV 2009).

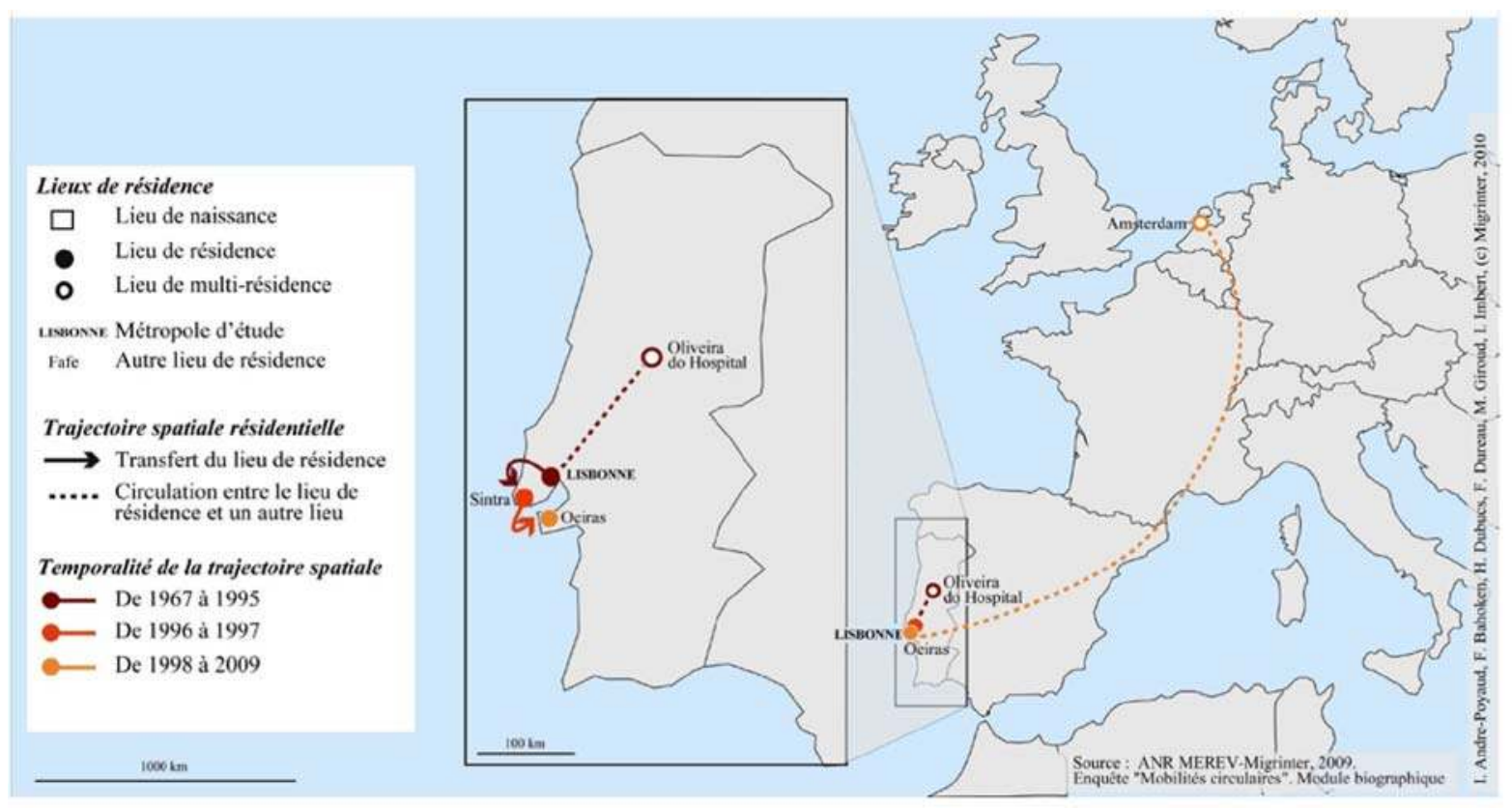

Sergio naît en 1967 à Lisbonne et y vit, chez ses parents, jusqu'en 1995. Pendant toute cette période, il connait une multi-résidence avec Oliveira do Hospital et passe de fréquentes vacances dans l'Algarve, habitude qu'il a encore aujourd'hui. Il se marie en 1996 et emménage avec sa femme dans un appartement qu'ils achètent à Sintra. Le couple déménage en 1998 à Oeiras, dans un appartement acheté, au moment où Sergio devient directeur financier de sa société (une multinationale du jouet), huit ans après avoir commencé à y travailler comme comptable. Le couple a un enfant en 1999. Les circulations européennes de Sergio s'intensifient au fur et à mesure qu'augmentent ses responsabilités professionnelles. De 2002 à 2004 il est birésident entre Oeiras et Amsterdam.

Nouvel entretien réalisé en 2010 : à partir de 2010, il passe 15 jours par mois à Barcelone et s'apprête à s'y installer avec sa famille pour un ou deux ans.

Trajectoire résidentielle de Sergio (enquête MEREV 2009) 
Annexes n8 : Extraits du questionnaire ENTD 2007.

Figure ${ }^{\circ} 1$.

Occupation du logement

\begin{tabular}{|c|c|}
\hline $\begin{array}{l}\text { TYPOLOG Type d'occupation du logement } \\
\text { (0. non (membre du ménage vivant ailleurs)) } \\
\text { 1. toute l'année ou presque } \\
\text { 2. plutôt les week-ends ou les vacances } \\
\text { 3. plutōt en semaine } \\
\text { 4. quelques mois dans Pannée } \\
\text { 5. plus rarement }\end{array}$ & $\begin{array}{l}\text { TYPLOGCO Type de logement collectif } \\
\text { 1. en caseme, en camp } \\
\text { 2. en intemat } \\
\text { 3. en cité universitaire ou dans un foyer détudiants } \\
\text { 4. dans un foyer de jeunes travailleurs } \\
\text { 5. dans un établissement pénitentiaire } \\
\text { 6. en sanatorium, dans un établissement de soins ou un hôpital } \\
\text { 7. dans une maison de retraite ou un hospice } \\
\text { 8. sur un chanter temporaire de travaux publics }\end{array}$ \\
\hline
\end{tabular}

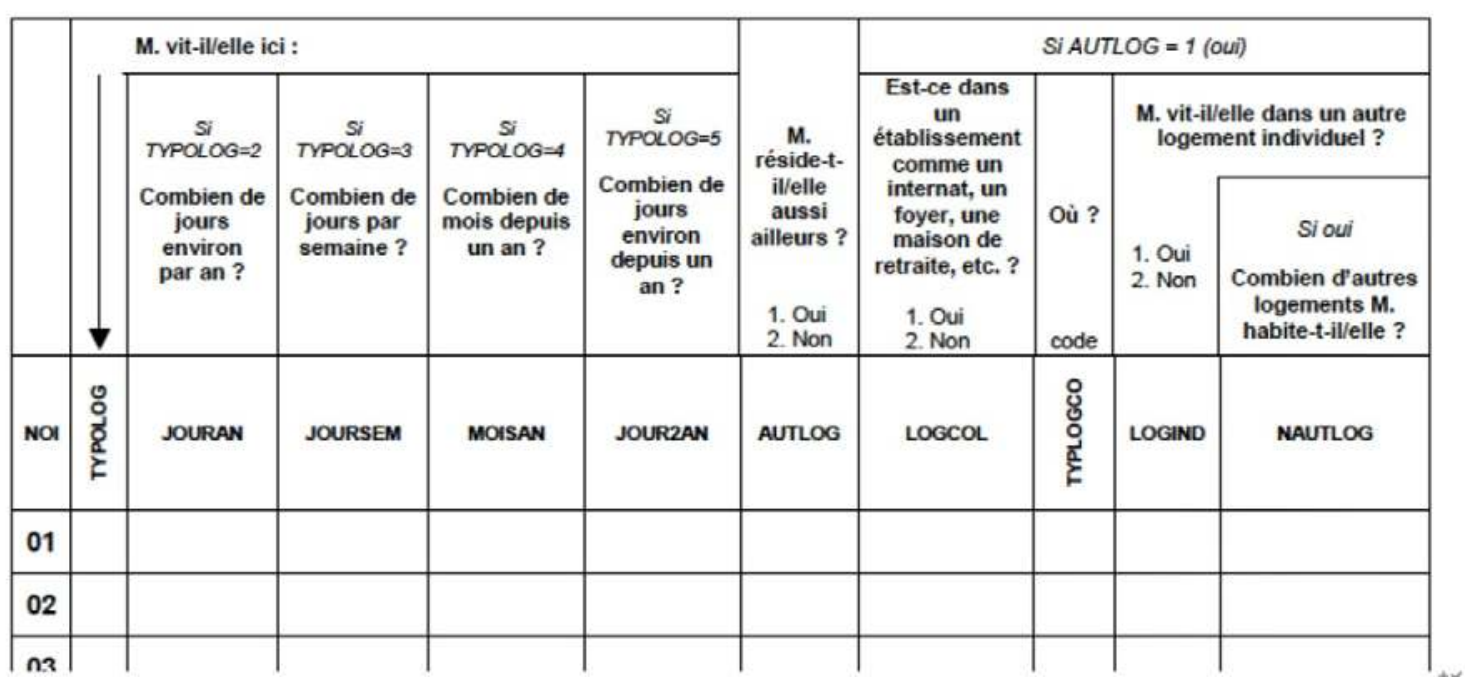

Figure $n^{\circ} 2$.

Si un habitant du logement habite aussi un autre logement (NAUTLOG > 0)

\section{Autres logements}

Vous m'avez dit qu'une ou plusieurs personnes habitant ici vivai(en)t aussi ailleurs. Parlons du premier de ces autres logements. Pour cela, comment voulez-vous le désigner ?

TYPOLOGD Type d'occupation du logement

1. Toute l'année ou presque

2. Plutôt les week-ends ou les vacances

3. Plutôt en semaine

4. Quelques mois dans l'année

5. Plus rarement

\section{CATLOGAD Catégorie du logement au sens du}

recensement

1. Résidence principale

2. Logement utilisé occasionnellement

3. Résidence secondaire ou de vacances

9. Ne sait pas

| Nom du logement

Le logement est-il

1. En France (y-compris DOM)?

2. Ailleurs?

Qui parmi les habitants du logement où nous sommes vit dans le logement? Cocher QUILOG pour les personnes

concermées. Pour chacune d'elles:

$M$. vit-il dans le

logement.

Le logement est-il aussi habité par des personnes qui ne vivent pas ici?

1. Oui

2. Non

9. Ne sait pas

Au total, combien de personnes viventelles dans le logement NOMLOG ?

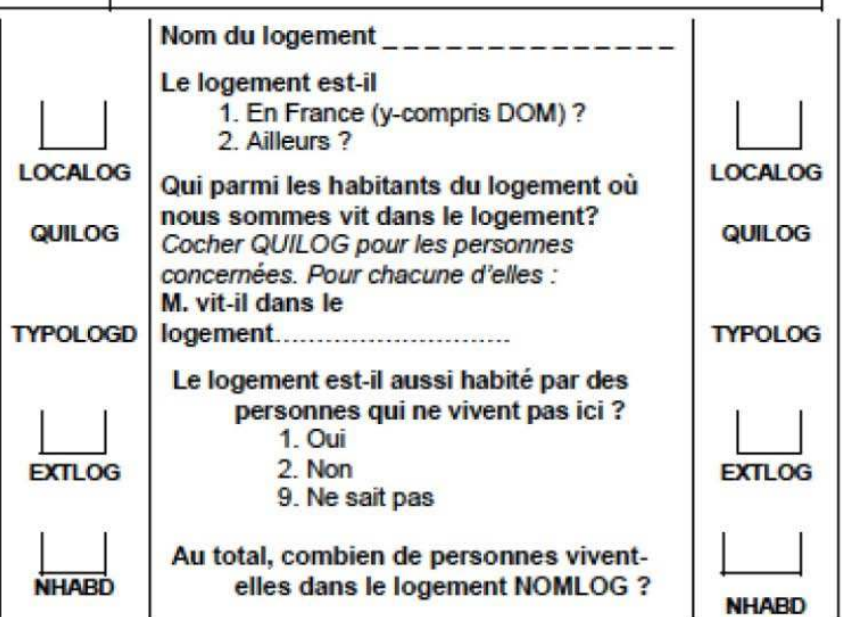


Figure $\mathbf{n}^{\circ} 3$.

\begin{tabular}{|c|c|c|c|c|c|c|c|c|c|c|c|c|c|}
\hline & \multicolumn{5}{|c|}{$\begin{array}{l}\text { POUR LA PREMIERE PERSONNE } \\
\text { DE CHAQUE MENAGE (BS) } \\
\text { Le logement est-il } \\
\text { 1. Un logement dont M. est Poccupant } \\
\text { principal ? } \\
\text { 2. Un logement oủ l'occupant principal est un } \\
\text { autre parent, un(e) ami(e)? } \\
\text { M. fait-il, dans la vie courante, budget } \\
\text { commun avec tout ou partie de ces } \\
\text { personnes ? } \\
\text { 1. Oui } \\
\text { 2. Non } \\
\text { 9. Ne sait pas } \\
\text { Pour le ménage de M., le logement est-il : } \\
\text { Pour le ménage de M., le logement est : } \\
\text { Une personne habilitée à répondre à } \\
\text { l'enquête est-elle susceptible d'être } \\
\text { contactée dans le logement avant (date de } \\
\text { fin de la collecte)? } \\
\text { 1. Oui } \\
\text { 2. Non } \\
\text { 9. Ne sait pas }\end{array}$} & \begin{tabular}{|l|} 
TLOGIND \\
UVLOG \\
CATLOGAD \\
CONTACTD
\end{tabular} & \multicolumn{5}{|c|}{$\begin{array}{l}\text { POUR LA PREMIERE PERSONNE } \\
\text { DE CHAQUE MENAGE (BS) } \\
\text { Le logement est-il } \\
\text { 1. Un logement dont M. est loccupant } \\
\text { principal? } \\
\text { 2. Un logement où l'occupant principal est un } \\
\text { autre parent, un(e) ami(e)? } \\
\text { M. fait-il, dans la vie courante, budget } \\
\text { commun avec tout ou partie de ces } \\
\text { personnes? } \\
\text { 1. Oui } \\
\text { 2. Non } \\
\text { 9. Ne sait pas } \\
\text { Pour le ménage de M., le logement est-il : } \\
\text { Pour le ménage de M., le logement est : } \\
\text { Une personne habilitée à répondre à } \\
\text { l'enquête est-elle susceptible d'être } \\
\text { contactée dans le logement avant (date } \\
\text { de fin de la collecte)? } \\
\text { 1. Oui } \\
\text { 2. Non } \\
\text { 9. Ne sait pas }\end{array}$} & \multirow[t]{2}{*}{ 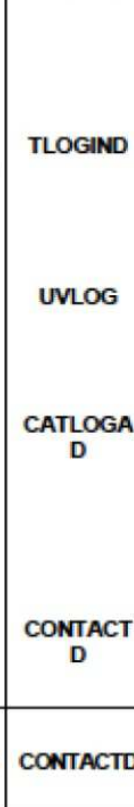 } \\
\hline NOI & BS & $\begin{array}{l}8 \\
\frac{9}{5} \\
0\end{array}$ & TYPOLOGD & TLOGIND & $\begin{array}{l}\stackrel{9}{9} \\
3\end{array}$ & CATLOGAD & CONTACTD & $\begin{array}{l}8 \\
\frac{8}{5} \\
0\end{array}$ & TYPOLOGD & TLOGIND & ฏ & CATLOGAD & \\
\hline 01 & & & & & & & & & & & & & \\
\hline 02 & & & & & & & & & & & & & \\
\hline 03 & & & & & & & & & & & & & \\
\hline 04 & & & & & & & & & & & & & \\
\hline 05 & & & & & & & & & & & & & \\
\hline 06 & & & & & & & & & & & & & \\
\hline 07 & & & & & & & & & & & & & \\
\hline 08 & & & & & & & & & & & & & \\
\hline 09 & & & & & & & & & & & & & \\
\hline 10 & & & & & & & & & & & & & \\
\hline
\end{tabular}

